Open Access

\title{
Comparative transcriptomics analysis reveals difference of key gene expression between banana and plantain in response to cold stress
}

Qiao-Song Yang ${ }^{1,2}$, Jie Gao ${ }^{1,2,3}$, Wei-Di He $e^{1,2,4}$, Tong-Xin Dou ${ }^{1,2,3}$, Li-Jie Ding ${ }^{1,2,3}$, Jun-Hua Wu ${ }^{1,2,3}$, Chun-Yu Li ${ }^{1,2}$, Xin-Xiang Peng ${ }^{3}$, Sheng Zhang ${ }^{5}$ and Gan-Jun $\mathrm{Yi}^{1,2^{*}}$

\begin{abstract}
Background: Banana and plantain (Musa spp.) comprise an important part of diets for millions of people around the globe. Low temperature is one of the key environmental stresses which greatly affects the global banana production. To understand the molecular mechanism of the cold-tolerance in plantain we used RNA-Seq based comparative transcriptomics analyses for both cold-sensitive banana and cold-tolerant plantain subjected to the cold stress for 0,3 and $6 \mathrm{~h}$.

Results: The cold-response genes at early stage are identified and grouped in both species by GO analysis. The results show that 10 and 68 differentially expressed genes (DEGs) are identified for 3 and $6 \mathrm{~h}$ of cold stress respectively in plantain, while 40 and 238 DEGs are identified respectively in banana. GO classification analyses show that the majority of DEGs identified in both banana and plantain belong to 11 categories including regulation of transcription, response to stress signal transduction, etc. A similar profile for 28 DEGs was found in both banana and plantain for $6 \mathrm{~h}$ of cold stress, suggesting both share some common adaptation processes in response to cold stress. There are 17 DEGs found uniquely in cold-tolerance plantain, which were involved in signal transduction, abiotic stress, copper ion equilibrium, photosynthesis and photorespiration, sugar stimulation, protein modifications etc. Twelve early responsive genes including ICEI and MYBS3 were selected and further assessed and confirmed by qPCR in the extended time course experiments $(0,3,6,24$ and $48 \mathrm{~h})$, which revealed significant expression difference of key genes in response to cold stress, especially ICE1 and MYBS3 between cold-sensitive banana and cold-tolerant plantain.

Conclusions: We found that the cold-tolerance pathway appears selectively activated by regulation of ICE1 and MYBS3 expression in plantain under different stages of cold stress. We conclude that the rapid activation and selective induction of ICE1 and MYBS3 cold tolerance pathways in plantain, along with expression of other cold-specific genes, may be one of the main reasons that plantain has higher cold resistance than banana.
\end{abstract}

Keywords: Comparative transcriptome analysis, Cold tolerance, Banana, Plantain, ICE1, MYBS3 pathways

\footnotetext{
* Correspondence: yiganjun@vip.163.com

${ }^{1}$ Institute of Fruit Tree Research, Guangdong Academy of Agricultural

Sciences, 80 Dafeng 2nd street, Tianhe District, Guangzhou, Guangdong

Province 510640, China

${ }^{2}$ Key Laboratory of South Subtropical Fruit Biology and Genetic Resource

Utilization, Ministry of Agriculture, Guangzhou 510640, China

Full list of author information is available at the end of the article
}

\section{Biomed Central}

(c) 2015 Yang et al. This is an Open Access article distributed under the terms of the Creative Commons Attribution License (http://creativecommons.org/licenses/by/4.0), which permits unrestricted use, distribution, and reproduction in any medium, provided the original work is properly credited. The Creative Commons Public Domain Dedication waiver (http:// creativecommons.org/publicdomain/zero/1.0/) applies to the data made available in this article, unless otherwise stated. 


\section{Background}

Low temperature is one of the key environmental stresses that many plants have to cope with during their life cycle, which can influence growth, development, as well as the yield, quality, postharvest life, and geographic distribution of crop plants [1,2]. Cold stress can be classified as chilling $\left(0-15^{\circ} \mathrm{C}\right)$ and freezing $\left(<0^{\circ} \mathrm{C}\right)$ stresses. Generally, plants from temperate regions have the capacity to cold acclimate, that is, to develop increased freezing tolerance after being exposed to low, nonfreezing temperatures [3], but many important crops, such as rice, maize, soybean, cotton, and tomato, which originated in the tropics and subtropics, lack the cold acclimation mechanism and are sensitive to chilling stress [4]. Moreover, different varieties of the same species can also exhibit a high degree of genetic variability for cold tolerance [5-7]. In conventional crop cross-breeding, the cold-tolerant varieties are usually used as the main resource for increasing the cold tolerance of cultivars [8]. However, the lack of detailed knowledge of molecular mechanisms responsible for cold stress limits the potential for crop improvement. Investigation of gene expression profiles in response to cold stress will shed light on the sensing and regulatory networks for cold acclimation in plants and provide an effective approach to select targeted candidate genes for manipulation and/or cross-breeding of agronomic plants [8].

Musa spp. (including Banana and Plantain), which originated in the tropics are giant perennial herbaceous monocots. They are vital staple food in many African countries and the most popular fruit in industrialized countries [9]. Musa spp. exhibits a high degree of genetic variability for cold tolerance, with banana (Musa spp. Cavendish; AAA Group) being more cold sensitive than plantain (Musa spp. Dajiao; ABB Group). When the temperature drops to $8^{\circ} \mathrm{C}$, banana growth is arrested, injury is inflicted [10], and irreversible damage often occurs with temperatures below $12^{\circ} \mathrm{C}$ [11]. In milder cases of cold injury, the fruit can be harvested, but its ripening process is abnormal. In contrast to the Cavendish "dessert" banana, the plantain species, Dajiao has superior cold tolerance, enabling it to tolerate temperatures of $0-4^{\circ} \mathrm{C}$ [12], and has been proposed as a potential germplasm resource of cold tolerance in banana breeding [10].

Although Musa spp. appear to lack a cold acclimation mechanism, transgenic plantain constitutively overexpressing the Arabidopsis transcription factor DREB1B/ $C B F 1$ becomes highly tolerant to cold by increasing SOD activities, decreasing MDA content and the electrolyte leakage rate. Meanwhile the growth rates of these transgenic plants are severely retarded under normal growth conditions [13]. In the herbaceous monocot rice (Oryza sativa), which also originated in the tropics, a novel MYBS3-dependent pathway has recently been identified as essential for cold tolerance. MYBS3 was found to repress the CBF-dependent cold signaling pathway. Molecular evidence indicates that the sequential expression of $C B F$ and $M Y B S 3$ provides two complementary mechanisms for conferring cold tolerance, with the $C B F$-mediated process initiating the immediate cold shock response and the MYBS3-mediated system adjusting the long-term cold adaptation in rice [14]. Recently, a new quantitative trait locus COLD1 was identified, which interacts with $\mathrm{G}$ protein to activate the $\mathrm{Ca}^{2+}$ channel for low temperature sensing that confers chilling tolerance in japonica rice, compared with cold-sensitive indica rice. Overexpression of COLD $1^{\text {jap }}$ significantly enhances chilling tolerance, whereas rice lines with deficiency or downregulation of COLD1 $1^{\text {jap }}$ are sensitive to cold stress [15]. In the past decade, some physiological responses to cold stress were analyzed, comparatively, between banana and plantain [16], and another cold-resistance related plantain $M p R C I$ (Rare cold-induced gene) has been identified, that enhances low temperature resistance when heterologously expressed in transgenic tobacco [17].

Through the traditional map-based cloning and transgenic methods, one can identify some key stress-related genes. But it's generally tedious and time-consuming, especially for fruit trees which have a relatively long juvenile phase. In recent years, advances in novel highthroughput sequencing technologies, such as Solexa/ Illumina RNA-Seq (RNA sequencing) and digital gene expression (DGE) has provided an opportunity to explore cold resistance and signaling-associated genes in different species by denovo assembly or mapping, facilitating rapid identification and analysis of many transcriptomes [18]. Transcriptome research has become an effective means to investigate the gene expression patterns of fruit trees in the growth and development process and under various stresses, which has been reported in tropical and subtropical fruit trees, such as litchi [19,20], mango [21], papaya [22], citrus [23] and grape [24] etc.

Our quantitative proteomic analysis reveals that molecular mechanisms for the higher cold resistance found in plantain are associated with increased redox potential characterized by adapted ROS scavenging capability, reduced ROS production, decreased lipid peroxidation, and cell wall stabilization [10]. Gene expression under cold stress is very sensitive and it depends on the species, the temperature and the length of exposure to low temperature [5-7]. Although several important clues are suggested from intensive proteomic research of coldtolerance plantain and comparing those results with functional gene analysis conducted in other plants, a comprehensive differential transcriptome analysis in response to cold stress between banana and plantain has not been reported and remains unknown.

In this study, plantain collected from a subtropical region of China with high cold-tolerance was used to 
investigate responses to cold stress at the transcriptional level. A cold-sensitive species, banana was examined as a control. A well-established whole genome transcriptome analysis method, based on RNA-Seq, and incorporating real time-PCR, was utilized to screen the differential transcripts associated with cold tolerance between banana and plantain. Our study provides a broad picture of the banana and plantain cold-responsive transcriptomes, with a new insight to cold-tolerance molecular mechanisms of plantain under cold stress.

\section{Results}

\section{Phenotypes and electrolyte leakage of banana and plantain to cold stress}

To accurately evaluate the tolerance of plantain, we compared the physiological changes of plantain versus banana in parallel during cold stress. A significant phenotypic difference is that the banana leaves drooped after $6 \mathrm{~h}$ of cold treatment and the second and third leaves from the top displayed severe necrosis and wilting symptoms after $48 \mathrm{~h}$ of cold treatment at $10^{\circ} \mathrm{C}$, while plantain leaves remained normal (Figure 1A and B). The relative leakage increased from 12.0 to $33.9 \%$ in banana leaves, and from 14.6 to $28.0 \%$ in plantain leaves respectively after $6 \mathrm{~h}$ of cold treatment. After $48 \mathrm{~h}$ of cold stress, the leakage of plantain leaves decreased to the basal level, but the leakage of banana leaves still increased and the damage was not alleviated (Figure 1C). These observations suggest that the seedlings of plantain leaves had adapted to the cold stress to some extent after $6 \mathrm{~h}$ of treatment and appeared to protect the membrane from further damage, suggesting that some important cold-tolerant genes, such as the early signal transduction genes of plantain, may work at the beginning $6 \mathrm{~h}$ of cold treatment. Both phenotypic response and relative leakage of plantain support the notion that plantain once again, has stronger cold tolerance than banana.

\section{RNA-Seq and alignment of unique reads to banana reference genome}

The raw data quality assessment of banana and plantain libraries with control and cold treatment are shown in Additional file 1: Table S1. For banana, after filtering out low quality reads, raw reads containing ' $\mathrm{N}$ ' and adaptor sequences, $12.66 \mathrm{M}$ to $16.96 \mathrm{M}$ clean reads were sequenced

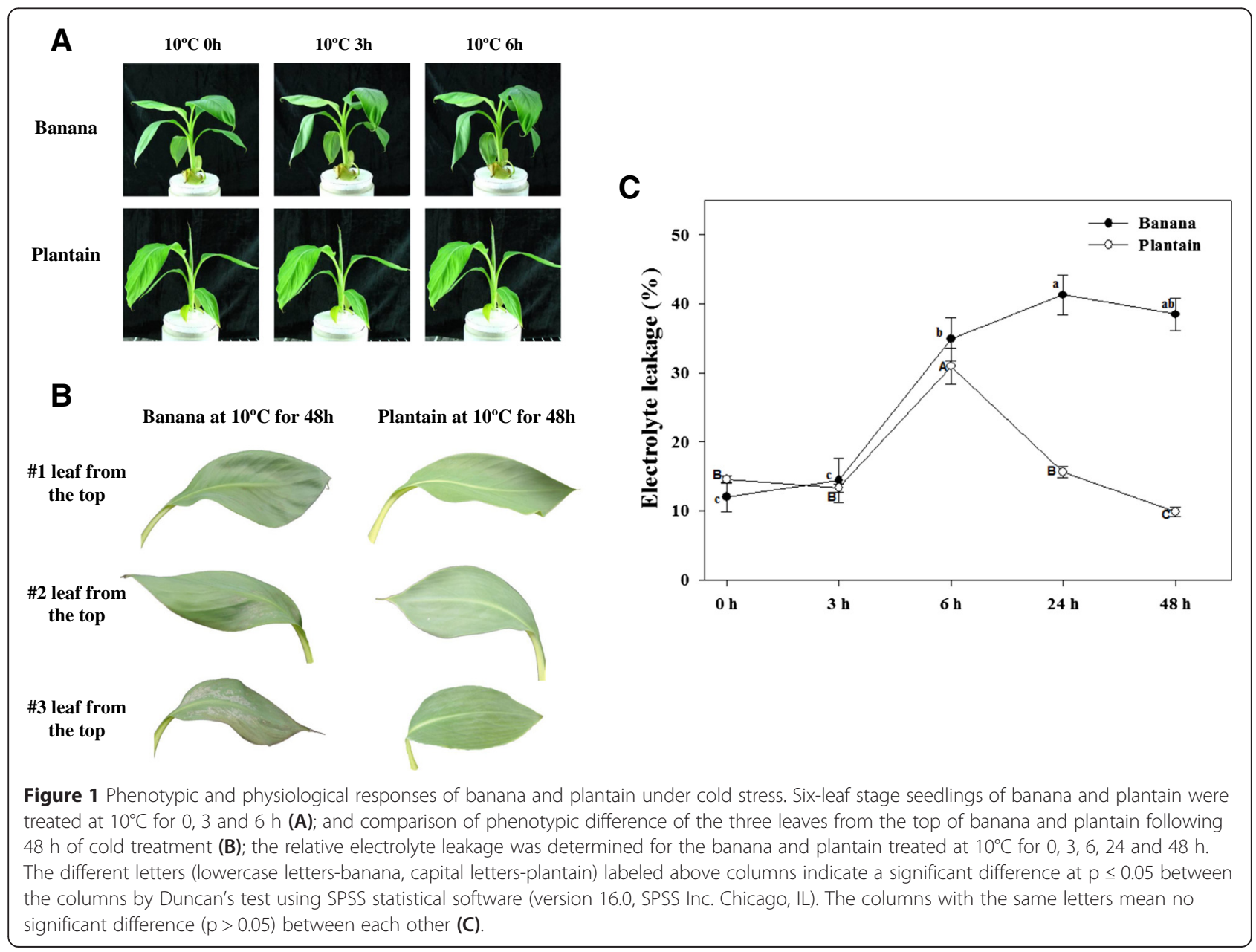


from four biological replicates of 0,3 and $6 \mathrm{~h}$ cold treated libraries, respectively (Table 1 ). Clean reads were aligned to banana reference genome database (doubled haploid Musa. acuminata genotype, A genome, http://bananagenome.cirad.fr/content/download-dh-pahang). About $60 \%$ Cavendish clean reads mapped to the reference genome. Multiple mapped clean reads in each library were excluded from further analysis. Finally a total of $5.14 \mathrm{M}$ to $7.55 \mathrm{M}$ uniquely mapped clean reads were used for subsequent analysis. The distribution of unique reads with chromosome '+/-' chain and splice/non-splice in each library were counted and are shown in Table 1.

For plantain, after filtering out low quality reads, raw reads containing ' $\mathrm{N}$ ' and adaptor sequences, $13.94 \mathrm{M}$ to 16.20 $\mathrm{M}$ clean reads were generated from four biological replicates of 0,3 and $6 \mathrm{~h}$ cold treated libraries, respectively (Table 1). Clean reads were aligning to banana reference genome database. There are also about $60 \%$ of plantain clean reads mapped to the reference genome. Finally a total of $5.04 \mathrm{M}$ to $5.94 \mathrm{M}$ uniquely mapped clean reads were used for further analysis. The distribution of unique reads with chromosome ' $+/-$ ' chain and splice/non-splice in each library were counted and are shown in Table 1.

\section{Differential expression profiling of cold stress between banana and plantain}

To comprehensively investigate the differences in gene expression between cold-sensitive banana and coldtolerant plantain in response to cold stress, we performed comparative transcriptome analysis using the aligned reads (above). After $3 \mathrm{~h}$ of cold stress, a total of 40 (33 upand 7 down-regulated), 10 (9 up- and 1 down-regulated) cold-responsive genes were identified in banana and plantain, respectively (Table 2, Figure 2, Additional file 2: Table S2 and Additional file 3: Table S3) with the threshold of 0.05 for Corrected $P$-value and 1 for $\log 2$ base of foldchange. Out of the 40 DEGs, 33 cold-responsive genes (26 up- and 7 down-regulated) were exclusively identified in banana, whereas 3 cold-responsive genes ( 2 up- and 1 down-regulated) were uniquely observed in plantain. The remaining 7 genes (all up-regulated) were commonly regulated by cold stress in both banana and plantain. After $6 \mathrm{~h}$ of cold stress, a total of 238 (195 up- and 43 downregulated), 68 (54 up- and 14 down-regulated) coldresponsive genes were identified in banana and plantain, respectively (Table 2 , Figure 2, Additional file 2: Table S2 and Additional file 3: Table S3). Out of the 238 DEGs, 188 cold-responsive genes (149 up- and 39 down-regulated) were exclusively identified in banana, whereas 18 coldresponsive genes ( 8 up- and 10 down-regulated) were uniquely observed in plantain and 50 genes (46 up- and 4 down-regulated) were commonly regulated by cold stress in both banana and plantain. The small number of coldresponsive genes identified in cold-tolerant plantain suggests that some inherent adaptation and regulation mechanisms may be attributed to the cold tolerance in plantain.

The DEGs with at least a 2-fold change are shown in Additional file 4: Table S4 and Table 3. After $3 \mathrm{~h}$ of cold

Table 1 Statistic analysis of Cavendish and Dajiao reads mapped to $M$. acuminate reference genome

\begin{tabular}{|c|c|c|c|c|c|c|}
\hline \multirow[t]{3}{*}{ Sample name } & \multicolumn{3}{|c|}{ Cavendish (Musa spp. Cavendish; AAA group) } & \multicolumn{3}{|c|}{ Dajiao (Musa spp. Dajiao; ABB group) } \\
\hline & $10^{\circ} \mathrm{C}$ & $10^{\circ} \mathrm{C}$ & $10^{\circ} \mathrm{C}$ & $10^{\circ} \mathrm{C}$ & $10^{\circ} \mathrm{C}$ & $10^{\circ} \mathrm{C}$ \\
\hline & $0 \mathrm{~h}$ replicates & $3 \mathrm{~h}$ replicates & $6 \mathrm{~h}$ replicates & $\mathrm{O} \mathrm{h}$ replicates & $3 \mathrm{~h}$ replicates & $6 \mathrm{~h}$ replicates \\
\hline Total reads $^{a}$ & $16.96 \pm 0.93$ & $14.54 \pm 0.03$ & $12.66 \pm 1.41$ & $13.94 \pm 1.51$ & $15.03 \pm 2.03$ & $16.20 \pm 0.33$ \\
\hline \multirow[t]{2}{*}{ Total mapped } & $10.30 \pm 0.78$ & $8.57 \pm 0.18$ & $7.59 \pm 0.79$ & $8.62 \pm 0.93$ & $9.27 \pm 1.16$ & $9.10 \pm 0.13$ \\
\hline & $(60.70 \pm 1.24 \%)$ & $(58.94 \pm 1.34 \%)$ & $(59.98 \pm 0.47 \%)$ & $(61.86 \pm 0.01 \%)$ & $(61.75 \pm 0.59 \%)$ & $(56.20 \pm 0.31 \%)$ \\
\hline \multirow[t]{2}{*}{ Multiple mapped } & $2.75 \pm 0.06$ & $3.12 \pm 0.37$ & $2.45 \pm 0.09$ & $3.20 \pm 0.32$ & $4.23 \pm 0.82$ & $3.17 \pm 1.19$ \\
\hline & $(16.22 \pm 0.52 \%)$ & $(21.44 \pm 2.50 \%)$ & $(19.46 \pm 1.50 \%)$ & $(23.31 \pm 4.79 \%)$ & $(28.02 \pm 1.65 \%)$ & $(19.67 \pm 7.75 \%)$ \\
\hline \multirow[t]{2}{*}{ Uniquely mapped } & $7.55 \pm 0.71$ & $5.45 \pm 0.55$ & $5.14 \pm 0.70$ & $5.43 \pm 1.25$ & $5.04 \pm 0.35$ & $5.94 \pm 1.32$ \\
\hline & $(44.48 \pm 1.76 \%)$ & $(37.51 \pm 3.84 \%)$ & $(40.53 \pm 1.02 \%)$ & $(38.55 \pm 4.80 \%)$ & $(33.74 \pm 2.23 \%)$ & $(36.54 \pm 7.44 \%)$ \\
\hline \multirow[t]{2}{*}{ Reads map to ' + ' } & $1.83 \pm 0.22$ & $1.33 \pm 0.11$ & $1.18 \pm 0.07$ & $1.25 \pm 0.18$ & $1.24 \pm 0.10$ & $1.46 \pm 0.20$ \\
\hline & $(10.75 \pm 0.70 \%)$ & $(9.17 \pm 0.76 \%)$ & $(9.34 \pm 0.52 \%)$ & $(8.94 \pm 0.32 \%)$ & $(8.33 \pm 0.46 \%)$ & $(9.02 \pm 1.04 \%)$ \\
\hline \multirow[t]{2}{*}{ Reads map to '-' } & $5.73 \pm 0.49$ & $4.12 \pm 0.44$ & $3.97 \pm 0.64$ & $4.18 \pm 1.07$ & $3.79 \pm 0.25$ & $4.47 \pm 1.12$ \\
\hline & $(33.7 \pm 1.06 \%)$ & $(28.34 \pm 3.08 \%)$ & $(31.19 \pm 1.54 \%)$ & $(29.61 \pm 4.47 \%)$ & $(25.41 \pm 1.78 \%)$ & $(27.52 \pm 6.40 \%)$ \\
\hline \multirow[t]{2}{*}{ Non-splice reads } & $7.10 \pm 0.64$ & $5.16 \pm 0.52$ & $4.92 \pm 0.67$ & $5.19 \pm 1.17$ & $4.83 \pm 0.32$ & $5.62 \pm 1.13$ \\
\hline & $(41.79 \pm 1.50 \%)$ & $(35.51 \pm 3.65 \%)$ & $(38.79 \pm 0.99 \%)$ & $(36.86 \pm 4.41 \%)$ & $(32.37 \pm 2.23 \%)$ & $(34.59 \pm 6.30 \%)$ \\
\hline \multirow[t]{2}{*}{ Splice reads } & $0.46 \pm 0.07$ & $0.29 \pm 0.027$ & $0.22 \pm 0.029$ & $0.24 \pm 0.08$ & $0.21 \pm 0.03$ & $0.32 \pm 0.19$ \\
\hline & $(2.69 \pm 0.27 \%)$ & $(2.00 \pm 0.18 \%)$ & $(1.75 \pm 0.029 \%)$ & $(1.69 \pm 0.39 \%)$ & $(1.38 \pm 0.00 \%)$ & $(1.95 \pm 1.13 \%)$ \\
\hline
\end{tabular}

${ }^{a}$ Total reads: clean data obtained after filtering the reads containing adapter, poly- $\mathrm{N}$ and low quality reads from raw data. (Unit: Million reads). 
Table 2 Differentially expressed genes (DEGs) in Cavendish and Dajiao during cold treatment for 3 and 6 hours

\begin{tabular}{lllllll}
\hline & Cavendish & Unique in Cavendish & Dajiao & Unique in Dajiao & Common in both \\
\hline DEGs at 3 h & Total & 40 & 33 & 10 & 3 & 7 \\
& Up-regulated & 33 & 26 & 9 & 2 & 7 \\
& Down-regulated & 7 & 7 & 1 & 1 & 0 \\
\multirow{2}{*}{ DEGs at 6 h } & Total & 238 & 188 & 68 & 18 & 50 \\
& Up-regulated & 195 & 149 & 54 & 8 & 46 \\
& Down-regulated & 43 & 39 & 14 & 10 & 4 \\
\hline
\end{tabular}

stress, 7 up-regulated genes commonly identified in both banana and plantain, include GSMUA_Achr9G07610_001 (Probable cytosolic iron-sulfur protein assembly protein 1), GSMUA_Achr6G32910_001 (Zinc finger CCCH domaincontaining protein 33), GSMUA_Achr2G13410_001 (Zinc finger protein 1), GSMUA_Achr6G25670_001 (U-box domain- containing protein 25), GSMUA_Achr7G26580_ 001(UDP-glucose 6-dehydrogenase), GSMUA_Achr7G 05900_001 (Dehydration-responsive element-binding protein 1D) and GSMUA_Achr3G23000_001 (Calcium-binding protein $\mathrm{KIC}$ ). Meanwhile, 2 up-regulated genes including GSMUA_Achr8G21550_001 (Ethylene insensitive 3-like 1 protein) and GSMUA_Achr3G05220_001 (Probable xyloglucan endotransglucosylase/ hydrolase protein 23) and 1 down-regulated gene: GSMUA_Achr3G04360_001 (Ethylene-responsive transcription factor RAP2-13) were significantly changed in plantain at 3 hours. Interestingly, the above 3 genes with the identical trend of changes were present in banana after $6 \mathrm{~h}$ of cold stress, suggesting that those 3 genes be specifically responsive to cold stress, and their early response maybe critical in the cold resistance. In addition, this timely delay of response to cold stress was also found in plantain. Out of 33 DEGs found in banana after $3 \mathrm{~h}$ of cold stress, 16 genes with the identical response profiling were present in plantain only after $6 \mathrm{~h}$ of cold stress. Those 16 genes include GSMUA_Achr 5G21050_001 (Ethylene-responsive transcription factor 1), GSMUA_Achr7G06910_001 (Ethylene-responsive transcription factor ERF105), GSMUA_Achr3G01650_001 (Ethylene responsive transcription factor RAP2-4), GSMUA_Achr 9G04780_001 (CBL-interacting serine/threonine protein kinase 11), GSMUA_Achr7G21780_001(NAC domaincontaining protein 68), GSMUA_Achr3G11070_001 (Zinc finger A20 and AN1 domain-containing stress associated protein 11), GSMUA_Achr10G22580_001 (E3 ubiquitinprotein ligase RING1), GSMUA_Achr 7G21070_001 (Probable mannan synthase 9), GSMUA_Achr10G25070_001 (Mitochondrial 2-oxoglutarate/malate carrier protein),

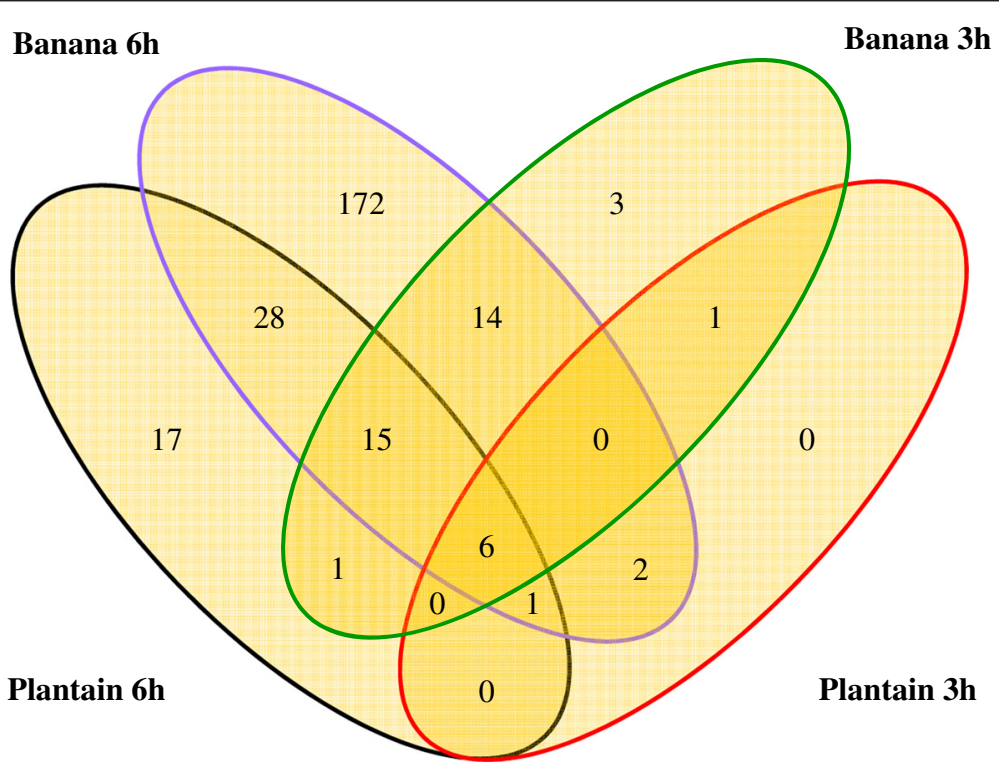

Figure 2 Venn diagram of differentially expressed genes (DEGs) identified for cold-sensitive banana and cold-tolerant plantain in response to cold stress. Green circle segment: the number of DEGs in banana under cold treatment at $10^{\circ} \mathrm{C}$ for $3 \mathrm{~h}$ with $0 \mathrm{~h}$ of banana as a control; Purple circle segment: the number of DEGs in banana under cold treatment at $10^{\circ} \mathrm{C}$ for $6 \mathrm{~h}, 0 \mathrm{~h}$ of banana as a control; Red circle segment: the number of DEGs in plantain under cold treatment at $10^{\circ} \mathrm{C}$ for $3 \mathrm{~h}, 0 \mathrm{~h}$ of plantain as a control; Black circle segment: the number of DEGs in plantain under cold treatment at $10^{\circ} \mathrm{C}$ for $6 \mathrm{~h}, 0 \mathrm{~h}$ of plantain as a control. 
Table 3 Primary functional classification on differentially expressed genes of Dajiao with fold changes $>$ or $<2$-fold

\begin{tabular}{|c|c|c|c|c|c|c|}
\hline Gene_id & $\begin{array}{l}\text { RPKM } \\
\text { Dajiao o h }\end{array}$ & $\begin{array}{l}\text { RPKM } \\
\text { Dajiao } 3 \mathrm{~h}\end{array}$ & $\begin{array}{l}\text { RPKM } \\
\text { Dajiao } 6 \mathrm{~h}\end{array}$ & $\begin{array}{l}\text { log2Fold } \\
\text { Change } 3 \mathrm{~h}\end{array}$ & $\begin{array}{l}\text { log2Fold } \\
\text { Change } 6 \mathrm{~h}\end{array}$ & Gene name \\
\hline \multicolumn{7}{|l|}{ Regulation of transcription } \\
\hline GSMUA_Achr8G25220_001 & 0.00 & & 74.93 & & $\operatorname{lnf}$ & $\begin{array}{l}\text { sp|Q9FDW1|MYB44_ARATH Transcription } \\
\text { factor MYB44 }\end{array}$ \\
\hline GSMUA_Achr6G15840_001 & 0.00 & & 244.92 & & Inf & $\begin{array}{l}\text { sp|Q9SKD9|WRK46_ARATH Probable WRKY } \\
\text { transcription factor } 46\end{array}$ \\
\hline GSMUA_Achr7G21780_001 & 3.92 & & 134.30 & & 5.08 & $\begin{array}{l}\text { sp|Q52QH4|NAC68_ORYSJ NAC } \\
\text { domain-containing protein } 68\end{array}$ \\
\hline GSMUA_Achr5G07590_001 & 3.49 & & 98.07 & & 4.79 & $\begin{array}{l}\text { sp|Q52QH4|NAC68_ORYSJ NAC } \\
\text { domain-containing protein } 68\end{array}$ \\
\hline GSMUA_Achr6G32330_001 & 13.18 & & 144.11 & & 3.35 & $\begin{array}{l}\text { sp|Q52QH4|NAC68_ORYSJ NAC } \\
\text { domain-containing protein } 68\end{array}$ \\
\hline GSMUA_Achr7G06910_001 & 92.07 & & 2467.93 & & 4.67 & $\begin{array}{l}\text { sp|Q8VY90|EF105_ARATH Ethylene-responsive } \\
\text { transcription factor ERF105 }\end{array}$ \\
\hline GSMUA_Achr5G21050_001 & 8.88 & & 112.43 & & 3.54 & $\begin{array}{l}\text { sp|Q40476|ERF1_TOBAC Ethylene-responsive } \\
\text { transcription factor } 1\end{array}$ \\
\hline GSMUA_Achr2G07300_001 & 89.60 & & 623.19 & & 2.69 & $\begin{array}{l}\text { sp|Q6K7E6|ERF1_ORYSJ Ethylene-responsive } \\
\text { transcription factor } 1\end{array}$ \\
\hline GSMUA_Achr8G06870_001 & 16.66 & & 182.33 & & 3.38 & $\begin{array}{l}\text { sp|Q9LW49|ERF4_NICSY Ethylene-responsive } \\
\text { transcription factor } 4\end{array}$ \\
\hline GSMUA_Achr4G33270_001 & 16.25 & & 187.60 & & 3.42 & $\begin{array}{l}\text { sp|Q9FE67|ERF80_ARATH Ethylene-responsive } \\
\text { transcription factor } 9\end{array}$ \\
\hline GSMUA_Achr3G01650_001 & 31.65 & & 237.25 & & 2.80 & $\begin{array}{l}\text { sp|Q8H1E4|RAP24_ARATH Ethylene-responsive } \\
\text { transcription factor RAP2-4 }\end{array}$ \\
\hline GSMUA_Achr7G11200_001 & 23.57 & & 226.28 & & 3.11 & sp|Q2R2W1|ADO3_ORYSJ Adagio-like protein 3 \\
\hline GSMUA_Achr8G21550_001 & 37.31 & 181.43 & 228.12 & 2.24 & 2.50 & $\begin{array}{l}\text { sp|Q9SLHO|EIL1_ARATH ETHYLENE INSENSITIVE } \\
\text { 3-like } 1 \text { protein }\end{array}$ \\
\hline GSMUA_Achr9G17080_001 & 176.04 & & 810.19 & & 2.09 & $\begin{array}{l}\text { sp|Q9SLHO|EIL1_ARATH ETHYLENE INSENSITIVE } \\
\text { 3-like } 1 \text { protein }\end{array}$ \\
\hline GSMUA_Achr6G32910_001 & 61.08 & 345.11 & 352.14 & 2.46 & 2.41 & $\begin{array}{l}\text { sp|Q688R3|C3H33_ORYSJ Zinc finger CCCH } \\
\text { domain-containing protein } 33\end{array}$ \\
\hline GSMUA_Achr11G02890_001 & 407.44 & & 97.21 & & -2.17 & $\begin{array}{l}\text { sp|A6MMN9|RPOA_DIOEL DNA-directed RNA } \\
\text { polymerase subunit alpha }\end{array}$ \\
\hline GSMUA_Achr3G04360_001 & 195.09 & 7.20 & & -4.82 & & $\begin{array}{l}\text { sp|Q9LM15|RA213_ARATH Ethylene-responsive } \\
\text { transcription factor RAP2-13 }\end{array}$ \\
\hline \multicolumn{7}{|l|}{ Response to stress } \\
\hline GSMUA_Achr7G05900_001 & 4.55 & 474.74 & 496.55 & 6.76 & 6.74 & $\begin{array}{l}\text { sp|Q9LWV3|DRE1D_ORYSJ } \\
\text { Dehydration-responsive element-binding } \\
\text { protein 1D }\end{array}$ \\
\hline GSMUA_Achr6G14750_001 & 1.82 & & 97.73 & & 5.72 & $\begin{array}{l}\text { sp|Q9SYM2|STHY_ARATH Probable salt } \\
\text { tolerance-like protein At1g78600 }\end{array}$ \\
\hline GSMUA_Achr5G07340_001 & 1.61 & & 80.13 & & 5.62 & $\begin{array}{l}\text { sp|Q10MX1|P2C32_ORYSJ Probable protein } \\
\text { phosphatase 2C } 32\end{array}$ \\
\hline GSMUA_Achr6G13020_001 & 9.58 & & 343.69 & & 4.98 & sp|Q8LGD5|MKS1_ARATH Protein MKS1 \\
\hline GSMUA_Achr2G13410_001 & 16.68 & 246.83 & 243.05 & 3.87 & 3.76 & sp|Q42430|ZFP1_WHEAT Zinc finger protein 1 \\
\hline GSMUA_Achr3G11070_001 & 208.08 & & 1667.24 & & 2.91 & $\begin{array}{l}\text { sp|Q84PD8|SAP11_ORYSJ Zinc finger A20 and AN1 } \\
\text { domain-containing stress-associated protein } 11\end{array}$ \\
\hline GSMUA_Achr10G22580_001 & 46.34 & & 325.56 & & 2.74 & $\begin{array}{l}\text { sp|POCH3O|RING1_GOSHI E3 ubiquitin-protein } \\
\text { ligase RING1 }\end{array}$ \\
\hline GSMUA_Achr7G27650_001 & 234.49 & & 1072.10 & & 2.10 & $\begin{array}{l}\text { sp|P49310|GRP1_SINAL Glycine-rich RNA-binding } \\
\text { protein GRP1A }\end{array}$ \\
\hline GSMUA_Achr7G00180_001 & 656.47 & & 2428.04 & & 1.76 & $\begin{array}{l}\text { sp|F6H7K5|THI42_VITVI Thiamine thiazole } \\
\text { synthase 2, chloroplastic }\end{array}$ \\
\hline
\end{tabular}


Table 3 Primary functional classification on differentially expressed genes of Dajiao with fold changes $>$ or $<\mathbf{2}$-fold (Continued)

\begin{tabular}{|c|c|c|c|c|c|c|}
\hline GSMUA_Achr8G20530_001 & 547.88 & & 145.12 & & -2.04 & $\begin{array}{l}\text { sp|P11432|ELI_PEA Early light-induced protein, } \\
\text { chloroplastic }\end{array}$ \\
\hline GSMUA_Achr7G20770_001 & 681.33 & & 129.38 & & -2.53 & sp|Q9MA41|CTL1_ARATH Chitinase-like protein 1 \\
\hline GSMUA_Achr3G03820_001 & 180.03 & & 20.24 & & -3.28 & $\begin{array}{l}\text { sp|Q00874|DR100_ARATH DNA-damage-repair/ } \\
\text { toleration protein DRT100 }\end{array}$ \\
\hline GSMUA_Achr1G17220_001 & 758.94 & & 19.48 & & -5.40 & $\begin{array}{l}\text { sp|Q9SW93|SCA_LILLO Stigma/stylar } \\
\text { cysteine-rich adhesin }\end{array}$ \\
\hline \multicolumn{7}{|l|}{ Protein modification } \\
\hline GSMUA_Achr9G08750_001 & 21.54 & & 308.06 & & 3.74 & $\begin{array}{l}\text { sp|Q9LT79|PUB25_ARATH U-box } \\
\text { domain-containing protein } 25\end{array}$ \\
\hline GSMUA_Achr6G25670_001 & 128.52 & 845.34 & 1563.67 & 2.68 & 3.48 & $\begin{array}{l}\text { sp|Q9LT79|PUB25_ARATH U-box } \\
\text { domain-containing protein } 25\end{array}$ \\
\hline GSMUA_Achr7G01430_001 & 52.60 & & 429.81 & & 2.95 & $\begin{array}{l}\text { sp|P05332|YP20_BACLI Uncharacterized } \\
\text { N-acetyltransferase p20 }\end{array}$ \\
\hline GSMUA_Achr2G13700_001 & 15.09 & & 110.79 & & 2.79 & $\begin{array}{l}\text { sp|Q10469|MGAT2_HUMAN Alpha-1, } \\
\text { 6-mannosyl-glycoprotein 2-beta-N- } \\
\text { acetylglucosaminyltransferase }\end{array}$ \\
\hline GSMUA_Achr6G18960_001 & 455.83 & & 2228.34 & & 2.19 & sp|POCH33|UBQ11_ARATH Polyubiquitin 11 \\
\hline \multicolumn{7}{|l|}{ Signal transduction } \\
\hline GSMUA_Achr9G04780_001 & 0.00 & & 111.31 & & Inf & $\begin{array}{l}\text { sp|O22932|CIPKB_ARATH CBL-interacting } \\
\text { serine/threonine-protein kinase } 11\end{array}$ \\
\hline GSMUA_Achr4G16550_001 & 13.05 & & 123.18 & & 3.19 & $\begin{array}{l}\text { sp|Q94BT2|AIR12_ARATH Auxin-induced in root } \\
\text { cultures protein } 12\end{array}$ \\
\hline GSMUA_Achr1G03980_001 & 58.82 & & 544.78 & & 3.15 & $\begin{array}{l}\text { sp|Q9ZPX9|KIC_ARATH Calcium-binding } \\
\text { protein KIC }\end{array}$ \\
\hline GSMUA_Achr3G23000_001 & 66.66 & 705.10 & & 3.41 & & $\begin{array}{l}\text { sp|Q9ZPX9|KIC_ARATH Calcium-binding } \\
\text { protein KIC }\end{array}$ \\
\hline GSMUA_Achr6G35120_001 & 299.39 & & 1905.08 & & 2.58 & $\begin{array}{l}\text { sp|Q9LNE7|CML7_ARATH Calmodulin-like } \\
\text { protein } 7\end{array}$ \\
\hline
\end{tabular}

Cell wall organization and

biogenesis

\begin{tabular}{|c|c|c|c|c|c|}
\hline GSMUA_Achr7G21070_001 & 12.21 & & 113.07 & 3.14 & $\begin{array}{l}\text { sp|Q67VS7|CSLA9_ORYSJ Probable mannan } \\
\text { synthase } 9\end{array}$ \\
\hline GSMUA_Achr6G05390_001 & 50.30 & & 294.18 & 2.46 & $\begin{array}{l}\text { sp|Q9LR44|U75B1_ARATH } \\
\text { UDP-glycosyltransferase 75B1 }\end{array}$ \\
\hline GSMUA_Achr3G05220_001 & 0.00 & 108.92 & Inf & & $\begin{array}{l}\text { sp|Q38910|XTH23_ARATH Probable xyloglucan } \\
\text { endotransglucosylase/hydrolase protein } 23\end{array}$ \\
\hline GSMUA_Achr3G26220_001 & 349.74 & & 19.19 & -4.32 & $\begin{array}{l}\text { sp|Q8LER3|XTH7_ARATH Probable xyloglucan } \\
\text { endotransglucosylase/hydrolase protein } 7\end{array}$ \\
\hline GSMUA_Achr7G22100_001 & 376.14 & & 6.12 & -6.08 & sp|Q0DHB7|EXPA4_ORYSJ Expansin-A4 \\
\hline \multicolumn{6}{|l|}{ Transport } \\
\hline GSMUA_Achr10G25070_001 & 42.74 & & 561.50 & 3.66 & $\begin{array}{l}\text { sp|Q9CR62|M2OM_MOUSE Mitochondrial } \\
\text { 2-oxoglutarate/malate carrier protein }\end{array}$ \\
\hline GSMUA_Achr7G16570_001 & 66.35 & & 290.42 & 2.03 & sp|P46032|PTR2_ARATH Peptide transporter PTR2 \\
\hline GSMUA_Achr1G22700_001 & 334.68 & & 1346.56 & 1.93 & sp|Q8VZ80|PLT5_ARATH Polyol transporter 5 \\
\hline \multicolumn{6}{|l|}{ Nucleosome assembly } \\
\hline GSMUA_Achr1G07160_001 & 64.61 & & 491.53 & 2.84 & sp|POCG89|H4_SOYBN Histone H4 \\
\hline GSMUA_Achr7G12120_001 & 443.53 & & 1635.08 & 1.76 & sp|Q9M5W4|H1_EUPES Histone H1 \\
\hline \multicolumn{6}{|l|}{ Cell redox homeostasis } \\
\hline GSMUA_Achr3G25160_001 & 0.00 & & 53.07 & $\operatorname{lnf}$ & $\begin{array}{l}\text { sp|Q84JR9|TTL4_ARATH TPR repeat-containing } \\
\text { thioredoxin TTL4 }\end{array}$ \\
\hline
\end{tabular}


Table 3 Primary functional classification on differentially expressed genes of Dajiao with fold changes $>$ or $<2$-fold (Continued)

\begin{tabular}{|c|c|c|c|c|c|c|}
\hline \multicolumn{7}{|l|}{ Iron-sulfur cluster assembly } \\
\hline GSMUA_Achr9G07610_001 & 0.00 & 343.42 & 275.72 & $\operatorname{lnf}$ & $\operatorname{lnf}$ & $\begin{array}{l}\text { sp|Q6FJ73|CIAO1_CANGA Probable cytosolic } \\
\text { iron-sulfur protein assembly protein } 1\end{array}$ \\
\hline \multicolumn{7}{|l|}{ Proteolysis } \\
\hline GSMUA_Achr11G04410_001 & 1.46 & & 120.93 & & 6.34 & $\begin{array}{l}\text { sp|Q766C3|NEP1_NEPGR Aspartic proteinase } \\
\text { nepenthesin-1 }\end{array}$ \\
\hline \multicolumn{7}{|l|}{ Oxidation reduction } \\
\hline GSMUA_Achr7G26580_001 & 83.33 & 882.49 & 1084.18 & 3.35 & 3.57 & $\begin{array}{l}\text { sp|Q96558|UGDH_SOYBN UDP-glucose } \\
\text { 6-dehydrogenase }\end{array}$ \\
\hline \multicolumn{7}{|l|}{ RNA modification } \\
\hline GSMUA_Achr4G11450_001 & 61.95 & & 416.78 & & 2.64 & $\begin{array}{l}\text { sp|Q9SKZ2|CAF1G_ARATH Probable } \\
\text { CCR4-associated factor } 1 \text { homolog } 7\end{array}$ \\
\hline \multicolumn{7}{|l|}{ Copper ion homeostasis } \\
\hline GSMUA_Achr3G16210_001 & 9685.89 & & 33776.00 & & 1.72 & $\begin{array}{l}\text { sp|Q40256|MT3_MUSAC Metallothionein-like } \\
\text { protein type } 3\end{array}$ \\
\hline \multicolumn{7}{|l|}{$\begin{array}{l}\text { Photosynthesis and } \\
\text { photorespiration }\end{array}$} \\
\hline GSMUA_Achr10G19930_001 & 210.99 & & 18.46 & & -3.62 & $\begin{array}{l}\text { sp|O24045|RBS_MUSAC Ribulose bisphosphate } \\
\text { carboxylase small chain, chloroplastic }\end{array}$ \\
\hline \multicolumn{7}{|l|}{$\begin{array}{l}\text { Response to sucrose } \\
\text { stimulus }\end{array}$} \\
\hline GSMUA_Achr11G11670_001 & 233.57 & & 3.50 & & -6.15 & $\begin{array}{l}\text { sp|Q9LZP9|CP122_ARATH Calvin cycle protein } \\
\text { CP12-2, chloroplastic }\end{array}$ \\
\hline \multicolumn{7}{|l|}{ Unknown } \\
\hline GSMUA_Achr2G22400_001 & 0.00 & & 59.28 & & $\operatorname{lnf}$ & Putative uncharacterized protein \\
\hline GSMUA_Achr10G24780_001 & 2.98 & & 136.75 & & 5.50 & Putative uncharacterized protein \\
\hline GSMUA_Achr6G10300_001 & 12.59 & & 230.91 & & 4.17 & Putative uncharacterized protein \\
\hline GSMUA_Achr2G22290_001 & 28.69 & & 317.86 & & 3.39 & Putative uncharacterized protein \\
\hline GSMUA_Achr5G01760_001 & 40.45 & & 432.42 & & 3.35 & Putative uncharacterized protein \\
\hline GSMUA_Achr3G28910_001 & 263.62 & & 1904.50 & & 2.75 & Putative uncharacterized protein \\
\hline GSMUA_Achr6G16830_001 & 61.64 & & 364.03 & & 2.45 & Putative uncharacterized protein \\
\hline GSMUA_Achr8G05250_001 & 163.34 & & 933.13 & & 2.41 & Putative uncharacterized protein \\
\hline GSMUA_Achr4G24680_001 & 409.42 & & 52.57 & & -3.08 & Putative uncharacterized protein \\
\hline GSMUA_Achr8G30530_001 & 245.64 & & 28.47 & & -3.20 & Putative uncharacterized protein \\
\hline GSMUA_Achr6G01490_001 & 158.51 & & 11.52 & & -3.90 & Putative uncharacterized protein \\
\hline GSMUA_Achr9G12290_001 & 65.93 & & 0.00 & & $-\operatorname{lnf}$ & Putative uncharacterized protein \\
\hline GSMUA_Achr9G26820_001 & 65.88 & & 0.00 & & $-\operatorname{Inf}$ & Putative uncharacterized protein \\
\hline
\end{tabular}

GSMUA_Achr7G00180_001 (Thiamine thiazole synthase 2), GSMUA_Achr6G13020_001 (Protein MKS1), GSMUA_ Achr10G24780_001 (Unknown) with up-regulation, and GSMUA_Achr11G02890_001 (DNA directed RNA polymerase subunit alpha), GSMUA_Achr7G22100_001 (Expansin-A4) and GSMUA_Achr 8G30530_001 (Unknown) with down-regulation. These delayed, expressed genes may be of importance to the adaptation of cold stress for sustainable cold tolerance in plantain.

There are 45 more DEGs being identified in plantain for $6 \mathrm{~h}$ of cold stress. Of which 28 genes include GSMUA Achr8G25220_001 (Transcription factor MYB44), GSMUA Achr 6G15840_001 (Probable WRKY transcription factor
46), GSMUA_Achr3G25160_001 (TPR repeat-containing thioredoxin TTL4), GSMUA_Achr11G04410_001 (Aspartic proteinase nepenthesin-1), GSMUA_Achr6G14750_ 001 (Probable salt tolerance-like protein), GSMUA_Achr 5G07340_001 (Probable protein phosphatase 2C), GSMUA_ Achr4G33270_001 (Ethylene-responsive transcription factor 9), GSMUA_Achr8G06870_001 (Ethylene responsive transcription factor 4), GSMUA_Achr7G11200_001 (Adagiolike protein 3), GSMUA_Achr7G01430_001 (Uncharacterized N-acetyltransferase p20), GSMUA_Achr 2 G13 700_001(Alpha-1,6-mannosyl-glycoprotein2-beta-N-acetylglucosaminyl transferase), GSMUA_Achr4G11450_001 (Probable CCR4-associated factor 1 homolog 7), GSMUA_ 
Achr6G16830_001 (Pentatricopeptide repeat-containing protein), GSMUA_Achr6G18960_001 (Polyubiquitin 11), GSMUA_Achr7G27650_001 (Glycine-rich RNA binding protein GRP1A), GSMUA_Achr1G22700_001 (Polyol transporter 5), GSMUA_Achr7G12120_001 (Histone H1), GSMUA_Achr3G03820_001(DNA-damage-repair/toleration protein DRT100), GSMUA_Achr2G22400_001 (Unknown), GSMUA_Achr6G10300_001 (Unknown), GSMUA_ Achr2G22290_001 (Unknown), GSMUA_ Achr5G01760_ 001 (Unknown), GSMUA_Achr 6G35120_001 (Unknown). A similar differential profile for those 28 genes was also found in banana for $6 \mathrm{~h}$ of cold stress, suggesting they share the similar adaptation mechanism to cold stress. The remaining 17 genes were differentially expressed only in plantain (no significant changes in banana). These genes include GSMUA_Achr4G16550_001 (Auxin-induced in root cultures protein 12), GSMUA_Achr1G07160_001 (Histone H4), GSMUA_Achr6 G05390_001(UDP-glycosyltransferase 75B1), GSMUA_Achr7G16570_001 (Peptide transporter PTR2), GSMUA_Achr3G16210_001 (Metallothionein-like protein type 3), GSMUA_Achr3G28910_001 (Unknown), GSMUA_Achr8G05250_001 (Unknown) with up-regulation, and GSMUA_Achr8G20530_001 (Early lightinduced protein), GSMUA_Achr7G20770_001 (Chitinaselike protein 1), GSMUA_Achr4G24680_001 (21 kDa protein), GSMUA_Achr10G19930_001 (Ribulose bisphosphate carboxylase small chain), GSMUA_ Achr3G26220_ 001 (Probable xyloglucan endotransglucosylase/hydrolase protein 7), GSMUA_Achr1G17220_001 (Stigma/stylar cysteine-rich adhesin), GSMUA_Achr 11G11670_001 (Calvin cycle protein CP12-2), GSMUA_Achr6G01490_ 001 (Unknown), GSMUA_Achr9G12290_001 (Unknown) and GSMUA_Achr9G26820_001 (Unknown) with down regulation. These 17 genes and their regulations appear likely attributed to the cold tolerance for plantain. Besides aforementioned 66 differential genes, 172 more DEGs were found only in banana for $6 \mathrm{~h}$ of cold stress, which are expected to be involved in cold stress for banana only.

\section{Functional classification of cold stress-related DEGs}

The functional classification of DEGs in banana and plantain was further examined to explore the pattern of transcriptome regulation that occurs during cold stress. Genes matching well-characterized proteins or proteins with putative functions were grouped using the gene ontology (GO) and summarized in Additional file 4: Table S4 and Table 3. The majority of DEGs identified in this study are in the categories regulation of transcription, response to stress, transport, protein modification, nucleosome assembly, cell wall organization and biogenesis, signal transduction, oxidation reduction, RNA modification, cell redox homeostasis, etc. No obvious difference of GO classification was observed for those DEGs from either banana (Additional file 4: Table S4) or plantain (Table 3), despite the fact that few DEGs in each GO category were identified in plantain than in banana.

\section{Validation of the DEGs by quantitative RT-PCR analysis}

Plantain leaves showed significant phenotypic difference from banana after $3 \mathrm{~h}$ cold treatment at $10^{\circ} \mathrm{C}$, we suspect that early response genes of plantain are closely associated with its cold resistance, thus 10 DEGs of plantain identified in this study after $3 \mathrm{~h}$ cold treatment and 2 critical cold-response homologous genes in arabidopsis ICE1 and rice MYBS3 were selected for quantitative RT-PCR analysis. Two additional extended time points for 24 and $48 \mathrm{~h}$ of cold stress were also measured in parallel with initial 0,3 and $6 \mathrm{~h}$ cold stressed samples in both banana and plantain. The primers of selected genes are listed in Additional file 5: Table S5. 25S ribosomal RNA gene was used as reference gene for data normalization according to Van den Berg et al. [25]. The quantitative RT-PCR results from banana and plantain are shown in Figure 3. The expression profiles of all 12 detected genes show the same trend and consistent results between the RT-PCR and the Solexa-sequencing methods. For 24 and 48 h of cold stress, 6 out of 10 DEGs displayed the same down regulation in both banana and plantain, including GSMUA_Achr7G05900_ 001 (Dehydration-responsive element-binding protein 1D), GSMUA_Achr9G07610_001 (Probable cytosolic iron-sulfur protein assembly protein 1), GSMUA_Achr6 G32910_001 (Zinc finger CCCH domain- containing protein 33), GSMUA_Achr7G26580_001 (UDP-glucose 6-dehydrogenase), GSMUA_Achr3G04360 _001 (Ethylene-responsive transcription factor RAP2-13), GSMUA_ Achr3G23000_001 (Calcium-binding protein KIC). However, 4 of them showed opposite changes between banana and plantain, including GSMUA_Achr6G25670_001 (U-box domain-containing protein25), GSMUA_Achr8G21550_001 (Ethylene insensitive 3- like 1 protein), GSMUA_ Achr2G 13410_001 (Zinc finger protein 1) and GSMUA_Achr 3G05220_001 (Probable xyloglucan endotransglucosylase/ hydrolase protein 23). Strikingly, there is a remarkable difference of ICE1 and MYBS3 expression profile between plantain and banana in response to cold stress. The ICE1 was significantly down-regulated (decreased by 4 -fold) under cold stress at early stage $(3 \mathrm{~h})$ in banana, but its decrease (by $\sim 5$-fold) didn't appear until at $24 \mathrm{~h}$ in plantain (Figure 3K). Although the MYBS3 was down-regulated similarly at early stage by 9 -fold and 4-fold in banana and plantain respectively, at $6 \mathrm{~h}$ of cold stress (Figure 3L), it can be recovered much quickly in plantain than banana. With the extended cold stress, the plantain MYBS3 was rapidly recovered to the level of $41 \%$ (at $24 \mathrm{~h}$ ) and $75 \%$ (at $48 \mathrm{~h}$ ), while the banana MYBS3 was further decreased to $4 \%$ at $24 \mathrm{~h}$ and then recovered to $24 \%$ at $48 \mathrm{~h}$ of cold stress. The considerably different expression profiles of 


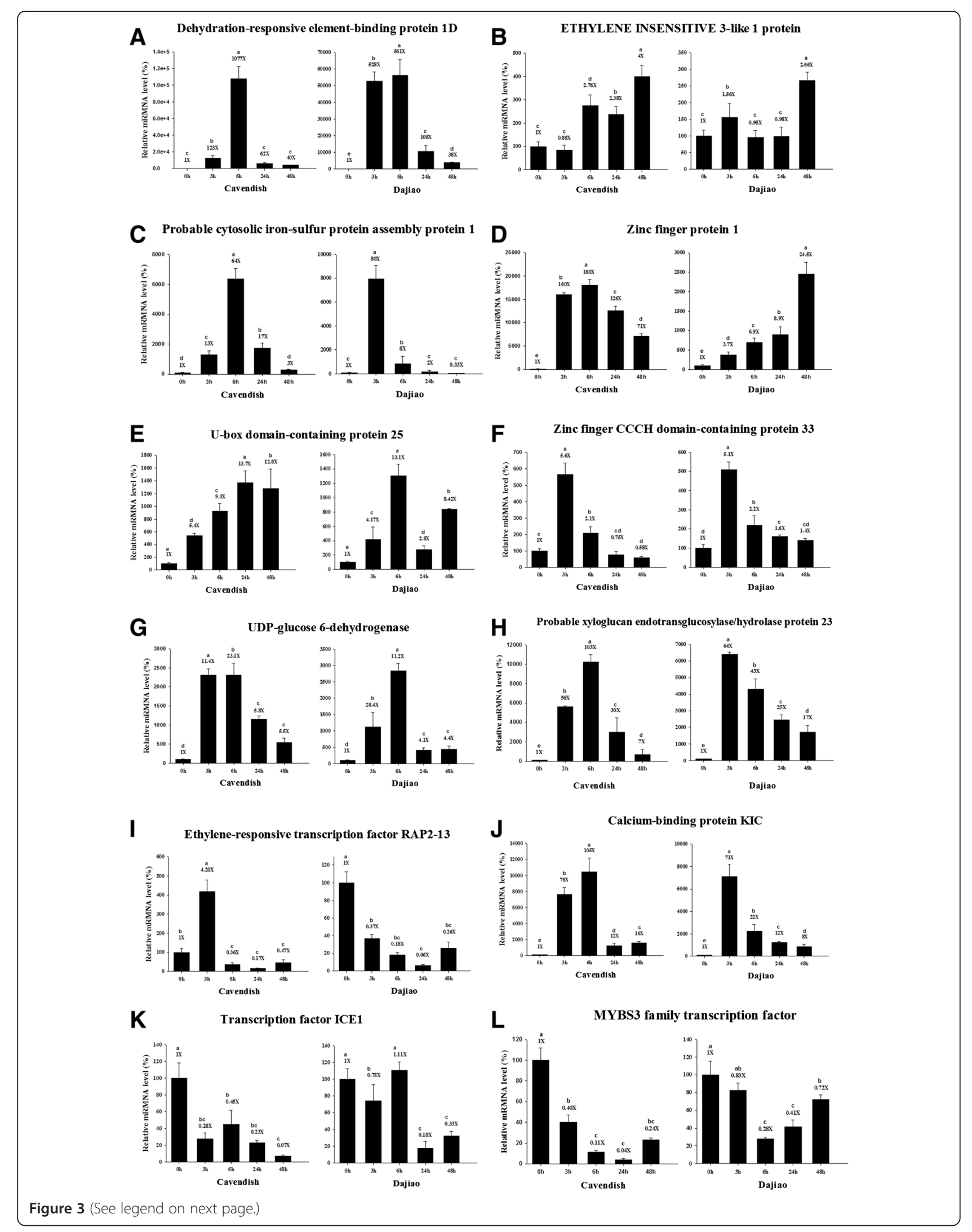


(See figure on previous page.)

Figure 3 Relative mRNA levels of 12 DEGs in banana and plantain seedlings were determined by quantitative RT-PCR analyses. Six-leaf stage seedlings were incubated at $10^{\circ} \mathrm{C}$ for the indicated time. Transcript abundances of genes encoding dehydration-responsive element-binding protein 1D (A), ethylene insensitive 3-like 1 protein (B), probable cytosolic iron-sulfur protein assembly protein 1 (C), zinc finger protein 1 (D), U-box domain- containing protein 25 (E), zinc finger CCCH domain-containing protein 33 (F), UDP-glucose 6-dehydrogenase (G), probable xyloglucan endotransglucosylase/hydrolase protein $23(\mathbf{H})$, ethylene-responsive transcription factor RAP2-13 (I), calcium-binding protein KIC (J), transcription factor ICE1 (K) and MYBS3 (L) from both banana and plantain were determined and compared across the time course of cold stress. Data represent means \pm SD in four replicates $(n=4)$. The different lowercase letters labeled above columns indicate a significant difference at $p \leq 0.05$ between the columns by Duncan's test using SPSS statistical software (version 16.0, SPSS Inc. Chicago, IL). The columns with the same letters mean no significant difference $(p>0.05)$ between each other.

the two important transcriptional factors with a remarkable time delayed response in banana versus plantain suggest that the specific time course-based expression of ICE1 and MYBS3 in plantain might be related to its cold tolerance.

\section{Discussion and conclusions}

In our previous study on the temporal responses of plantain to cold stress using quantitative proteomics analysis, we have revealed that antioxidation mechanisms contribute to cold tolerance in plantain at the global proteome level [10]. However, due to the limitation of current technologies in overcoming the issue of the wide dynamic range in proteomics samples, quantitative proteomics analysis often misses the detection of many low abundance proteins, yielding only $10-30 \%$ proteome coverage for any given non-model species. Obviously this will reduce the number of low abundance proteins identified during global functional studies. Given the above consideration and to focus on the molecular mechanisms involving low abundance genes on the cold-tolerance of plantain, a comparative transcriptomics analysis of cold-sensitive banana and cold-tolerant plantain was conducted by RNA-Seq and real time RTPCR with time course of cold treatment.

After $48 \mathrm{~h}$ of cold stress at $10^{\circ} \mathrm{C}$ for banana and plantain seedlings, both phenotype and electrolyte leakage analyses clearly indicate that plantain seedlings exhibited a more robust cold tolerance than banana seedlings (Figure 1). It is this phenotypic difference in the cold response between banana and plantain that provides an excellent model system allowing us to study differential gene expression in response to cold stress, as well as to elucidate the potential different cold responsive mechanisms between banana and plantain. RNA-Seq analysis shows that 10 and 68 DEGs are identified for $3 \mathrm{~h}$ and $6 \mathrm{~h}$ of cold stress respectively in plantain, while the equivalent DEGs are 40 and 238 being identified in banana (as shown in Figure 2), indicating banana is much more sensitive to cold stress than plantain. GO classification analysis shows the majority of DEGs identified in this study belong to the following 11 categories: regulation of transcription, response to stress, transport, protein modification, nucleosome assembly, cell wall organization and biogenesis, signal transduction, oxidation reduction, RNA modification, cell redox homeostasis, etc. Since there is no difference of $\mathrm{GO}$ classification found between banana (Additional file 4: Table S4) and plantain (Table 3), it suggests that cold stress appears to have a broad range of impacts on cellular activities. However, when we further performed the pathway analysis for some specific DEGs either found uniquely in plantain particularly or detected at variable cold stress time points in both banana and plantain, we found that many DEGs and their associated pathways are likely to be involved in cold-tolerance of plantain. Below we discuss intensively some of the important pathways and their possible mechanisms associated with the observed cold tolerance in plantain.

\section{ICE1 and MYBS3 pathways}

$I C E$ gene was initially identified and isolated from Arabidopsis thaliana. The function of AtICE1 is to activate gene expression of $\mathrm{C}$-repeat binding factor (CBF3) to enhance the cold resistance of Arabidopsis [26]. In Oryza sativa, three novel MYB proteins (MYBS1, MYBS2 and MYBS3) containing one highly conserved DNA-binding domain mediate sugar and hormone regulation by promoting gene expression of alpha-amylase [27]. Recent studies reveal that $M Y B S 3$ is also involved in adaptation of cold stress in rice. Compared to the ICE1, MYBS3 functions particularly in late stage of plant for adaptation of cold stress. For example, transgenic rice overexpressing MYBS3 can resist cold stress at $4^{\circ} \mathrm{C}$ for a week [14]. In the early stage of cold response, the transient activation of $\alpha A m y 3$ expression by $C B F$ allows hydrolysis of reserved starch to meet the immediate need for a carbon source and energy to combat the cold shock, while the subsequent suppression of $\alpha A m y 3$ expression by $M Y B S 3$ allows rice to conserve carbohydrates until regrowth is allowed at elevated temperatures [14]. In this study using RNA-Seq analysis for 0,3 , and 6 h of cold stressed samples, although both ICE1 and MYBS3 genes were detected, neither was identified as DEG. This is not surprising, because ICE1 requires activation, which is regulated by post-translational modifications via phosphorylation and sumoylation of the SIZ1 gene [28,29]. Thus, ICE1 is likely not regulated at the transcriptional level. Since $M Y B S 3$ plays a role in cold adaptation at late stage of plant 
growth, MYBS3 is expected not present in early time points from cold stressed samples. In fact, we did identify both genes by RNA-Seq analysis at a low level with relatively large variation across the 4 replicate samples. Given the well-known functions of both ICE1 and MYBS3 genes in plant resistance to cold stress, we decided to use quantitative RT-PCR for assessing their changes with the extended time points at 24 and $48 \mathrm{~h}$ of cold stress in this study. The results indicate that although there is no significant change of ICE1 in plantain at 3 and $6 \mathrm{~h}$ cold stress, both an upstream gene: GSMUA_Achr9P04380_ 001(MpSIZ1, 68\% homology with arabidopsis SIZ1) and a downstream gene: GSMUA_Achr7 G05900_001 (CBF) in an ICE1-CBF-COR pathway, were up-regulated (Additional file 6: Figure S1; Figure 3A). Since the ICE1-CBF-COR is a well-known pathway involved in plant cold-tolerance [30], the up-regulation of $C B F$ is therefore, expected to facilitate the cold-tolerant pathway. A 3.5-fold increase of COR47 protein found in our proteomics analysis of plantain for $6 \mathrm{~h}$ under cold stress at $8^{\circ} \mathrm{C}$ [10] supports this observation with the ICE1-CBF-COR pathway being activated in response to the cold stress in this study. Interestingly, at $24 \mathrm{~h}$ of cold stress, both plantain ICE1 and CBF were down regulated. Meanwhile, the MYBS3 gene had almost completely recovered (from early down-regulation in response to the cold stress), which may offset the down-regulated ICE1-CBF-COR pathway as a means of sustaining the cold resistance. In banana, however both ICE1 and MYBS3 were significantly decreased under cold stress. The $C B F$ response to cold stress in banana is significantly slower than that in plantain, until $6 \mathrm{~h}$ of cold stress, when the banana $C B F$ gene was up-regulated, only to go down again at $24 \mathrm{~h}$ of cold stress. Consistently, the time delayed response of MYBS3 gene to the cold stress was also observed in banana. However, at $48 \mathrm{~h}$ of cold stress, the banana MYBS3 gene appeared recover from its initial cold suppression. Based on the observation of slow response to the cold stress in banana for both ICE1 and MYBS3 as the two critical transcriptional factors responsible for early and late stage respectively against cold stress, we suspect that the relatively low expression of these two key genes along with their delayed response to the cold stress are among the main reasons for cold sensitive banana.

\section{Signals transduction}

As an important second messenger, $\mathrm{Ca}^{2+}$ plays a vital role in the plant cold-stress response. The concentration of $\mathrm{Ca}^{2+}$ inside the cell increases rapidly during cold stress, followed by a number of signals mediated by a series of protein phosphorylation cascades [31]. During rice domestication, an amino acid mutant of COLD1 from $\mathrm{Met}^{187} / \mathrm{Thr}^{187}$ to Lys ${ }^{187}$ in japonica cultivars was found to enhance cold tolerance partly because it facilitates the formation of an appropriate $\mathrm{Ca}^{2+}$ signal by increased $\mathrm{Ca}^{2+}$ concentration [15]. Interestingly, our study shows that most of the DEGs involving in signal transduction are indeed related to the calcium-dependent signal pathway, such as orthologs of $C I P K$ ( $C B L$-interacting protein kinase), KIC (Kinesin-like calmodulin binding protein) and $C M L$ (Calmodulin-like protein). All three genes in banana and plantain were up-regulated in response to cold stress. In addition, overexpression of OsMSR2, a novel rice calmodulin-like gene, improves resistance of drought and salt and increases $A B A$ sensitivity in Arabidopsis [32]. In this work, we found the dramatically increased expression of CML7 (GSMUA_Achr6G35120_001), an ortholog of OsMSR2, in response to cold stress in plantain but not in banana, suggesting that there is a more efficient response of the $\mathrm{Ca}^{2+}$ signal transduction pathway in plantain so that it can quickly and effectively regulate downstream signaling and gene expression in response to cold stress.

As a signal molecule, auxin plays an important function under various abiotic stress conditions [33]. Intriguingly, compared to the control group $(0 \mathrm{~h})$, the expression of GSMUA_Achr4G16550_001 (Auxin-induced in root cultures protein 12, AIR12) was increased by 9 fold in $6 \mathrm{~h}$ cold treatment in plantain, while no differential expression of the gene was detected in banana. On the plasma membrane, AIR12 is able to receive auxin signal to promote lateral root morphogenesis and participates in the decomposition of glucose [34], while it has been proven that the content of soluble sugar and acid can affect the plant's cold tolerance $[35,36]$. Our results indicate that the increased expression of AIR12 in plantain could facilitate the growth of plantain root and the content of sugar and acid, which might be attributed to the better resistance to cold.

\section{Transcription regulation}

In this study, we have identified 10 differentially expressed transcription factors in response to cold stress in banana and plantain, including $N A C, E R F$ (Ethylene response transcription factors), DREB (Dehydrationresponsive element-1binding protein), MYB, WRKY, C3H33, EIL1 (Ethylene insensitive 3-like 1 gene), ADO3 (Adagiolike gene 3), ZFP (Zinc finger protein) and $R P O A$ (DNA-directed RNA polymerase subunit alpha). NAC transcription factors were up-regulated in banana and plantain after $6 \mathrm{~h}$ cold stress, and over-expression of SNAC2 was reported to increase cold and salt resistance in rice [37], suggesting that NACs are likely to participate in cold response in banana and plantain. Effects of ethylene on plants under cold stress have recently been recognized, the ERF transcription factors which related to ethylene, were up-regulated in banana and plantain after $6 \mathrm{~h}$ cold stress. Overexpression of transcription factor TERF2/ $L e E R F 2$ in tobacco and tomato was reported to result in 
cold tolerance by facilitating ethylene biosynthesis [38], which supports the important role of $E R F$ in plant cold tolerance. $D R E B$ transcription factors, with a conservative $A P 2 / E R E B P$ domain, are involved in regulation of stressrelated gene in response to the external environment, through binding DRE CIS-acting elements [39]. Our data shows that overexpression of DREB1 occurs at $3 \mathrm{~h}$ of cold stress in plantain versus $6 \mathrm{~h}$ in banana, suggesting that the early response of $D R E B 1$ may be critical in cold-tolerant plantain. Due to a large number of $M Y B$ transcription factor found in plants such as Arabidopsis thaliana and Zea mays, an increased amount of research has been focused on its role in transcriptional regulation and its impact on a broad range of physiological functions, e.g., overexpression of rice MYB genes (OsMYB3R-2 and OsMYB4) enabled to significantly enhanced the cold tolerance of transgenic Arabidopsis thaliana [40]. In our work,we found GSMUA_Achr8G25220_001-a MYB transcription factor increased after $6 \mathrm{~h}$ cold stress while it was undetectable at $0 \mathrm{~h}$ in both banana and plantain, indicating that this gene is a cold-induced gene. The change of this $M Y B$ gene could affect downstream related genes. In Arabidopsis thaliana, the WRKY gene family contains 17 genes induced by cold stress, most of which are activated in its early stages $[41,42]$. After $6 \mathrm{~h}$ cold stress, the WRKY genes showed up-regulation, among them, GSMUA_Achr7G 05200_001 was overexpressed whereas it was undetectable at $0 \mathrm{~h}$, indicating it is also a cold-induced gene. In plantain, only one cold-induced WRKY gene-GSMUA_ Achr6G15840_001 was detected after 6 h of cold stress. However, its expression was much higher than that in banana, suggesting that this WRKY gene may contribute to the cold-resistance of plantain. It has been reported that zinc-finger proteins are involved in resistance to adversity in plant [43]. For example, GmZF1 from soybean was found to enhance the tolerance of Arabidopsis to cold stress by expression of cold-regulation genes in the transgenic Arabidopsis [44]. Strikingly, banana ZFP1 is not up-regulated until $24 \mathrm{~h}$ of cold stress and starts to decrease at $48 \mathrm{~h}$, while plantain $Z F P 1$ is constantly upregulated beyond $48 \mathrm{~h}$ of cold stress, suggesting ZFP1 may play a role in late stages of cold stress (Figure 3D). Expression of EIL1 in plantain reached $\sim 11$-fold higher induction at $3 \mathrm{~h}$ cold treatment and then decreased back to initial levels at $48 \mathrm{~h}$. In banana, the EIL1 was upregulated by $\sim 9$-fold at $6 \mathrm{~h}$ and further increased to $\sim 13$-fold at $48 \mathrm{~h}$ of cold treatment (Figure $3 \mathrm{~B}$ ). As a primary transcription factor in ethylene signal transduction, EIL regulates transcription of downstream genes to complete the ethylene response [45]. Given the higher expression of EIL at very early stages and its rapid decrease in response to cold stress in plantain, we suspect that rapid and early activation of the ethylene signal transduction pathway appears to play a critical role in the rapid response to cold stress allowing early protection in plantain.

\section{Genes associated with stress response}

Plants often activate similar pathways in response to different types of abiotic stress. In this cold stress study, we found quite a few genes related to stress response in both banana and plantain, such as STHY (Probable salt tolerance-like protein), P2C32 (Probable protein phosphatase $2 \mathrm{C} 32$ ), ubiquitin-protein ligase genes, THIL-2 (Thiamine thiazole synthase 2), GRP(Glycine-rich RNAbinding protein) and DRT100 (DNA-damage-repair/ toleration protein), etc. Those genes showed similar patterns of expression in response to cold treatment in banana and plantain, suggesting the stress response genes share some common response pathways in both species. However, it should be noted that some of the DEGs are located in the chloroplast of plantain only, such as ELIP, CTL1, SCA and MKS1. In Rhododendron catawbiens, expression of RcELIP (early light-induced protein) resulted in adaptive responses to cold and high light in winteradapted rhododendron leaves, suggesting a critical role of ELIP in protection of photosynthetic apparatus from these stresses [46]. In Arabidopsis thaliana, CTL1 (Chitinase-like protein) is involved in altering the architecture of the root system in response to multiple environmental conditions [47]. As an MAP kinase substrate, Arabidopsis MKS1 may be involved in MPK4-regulated activation of plant defense by coupling the kinase to specific WRKY transcription factors [48]. Due to the expression of ELIP, CTL1, SCA and MKS1 induced by cold treatment exclusively in plantain (not in banana), we believe that plantain possesses the highly superior aspects of photosynthesis, root architecture and plant defense regulated by MPK, which makes plantain broadly resist to abiotic stress resulting in a better cold tolerance.

\section{Other DEGs involved in functional regulations}

In addition to the above four categories, we found some DEGs in banana and plantain are also involved in protein/ RNA modification, cell wall composition and occurrence, transit, nucleosome assembly, cellular redox balance, ironsulfur cluster assembly, protein hydrolysis, oxidation and reduction, copper ion balance, photosynthesis, respiration and sugar stimulus response. But again some of the cold responsive DEGs are found exclusively in plantain, including MTP3 (a 3-fold increase at $6 \mathrm{hr}$ cold stress) involved in copper ion balance, $R B C S$ (a 10-fold decrease at $6 \mathrm{hr}$ ) functioning in photosynthesis and respiration in chloroplast, CP122 (an 80-fold decrease at $6 \mathrm{hr}$ ) involved in sugar stimulus response in chloroplast, and UBQ11 (a 5fold increase at $6 \mathrm{hr}$ ) functioning in protein modification. In yeast, PutMT2 (type 2 metallothionein-like protein in Puccinellia tenuiflora) has been verified to play a critical 
role in improving tolerance to metal and reactive oxygen species [49]. Thus the up-regulation of MTP3 would allow plantain for improved tolerance to the increased reactive oxygen species (ROS) due to the cold stress [10]. In plant, RBSC encodes the small subunit of Rubisco (Ribulose-1, 5-bisphosphate carboxylase/oxygenase) involved in the first major step of carbon fixation and the RBSC gene family functions to yield sufficient Rubisco content for leaf photosynthetic capacity [50]. As the expression of RBCS was highly repressed in plantain, we speculate that the protection mechanism is activated in response to cold treatment in chloroplast in order to decrease the production of ROS through the weakened photosynthesis. Arabidopsis CP12 forms a complex with GAPDH (Glyceraldehyde-3phosphate dehydrogenase) and PRK(Phosphoribulokinase) in regulating TCA cycle [51]. A dramatic repression of CP122 suggests that plantain must reduce the photosynthesis in response to cold stress. This is consistent with the decreased $R B C S$ but as another pathway for the selfprotection mechanism. $U B Q$ (poly-ubiquitin gene) is involved in protein hydrolysis in the ubiquitin-proteasome pathway. In order to compensate cells for ubiquitin molecules in signal transmission, expression of poly-ubiquitin gene is increased in response to stress [52]. Given the increased expression of $U B Q$ in response to cold treatment, we suspect that UBQ might be involved in stress response in plantain.

In summary,a global, comparative transcriptomic profile of banana and plantain in response to cold treatment has been intensively investigated by transcriptome sequencing $(0 \mathrm{~h}, 3 \mathrm{~h}$ and $6 \mathrm{~h})$ and quantitative RT-PCR $(0 \mathrm{~h}, 3 \mathrm{~h}, 6 \mathrm{~h}, 24 \mathrm{~h}$ and $48 \mathrm{~h})$. At the transcriptomic level, both plantain and banana share similar profiles for the majority of DEGs in time course response to cold treatment. Many of the DEGs have been reported and involved in the development of cold resistance or other stress resistance in plant, such as: CIPK, KIC, CML (involved in signal transduction), NAC, ERF, DREB, MYB, WRKY, C3H33, ADO3, RPOA (involved in transcriptional regulation), $S T H Y, P 2 C 32$, ubiquitin-protein ligase genes, THIL-2, GR and DRT100 (involved in stress response), etc. Despite the similar profiles of DEGs found in banana and plantain, several dozen DEGs involved in several different pathways were successfully identified and validated only in cold-tolerant plantain. For example, the cold-tolerant ICE1 and MYBS3 pathways were rapidly activated and switched in early $(3 \mathrm{~h})$ and late $(24 \mathrm{~h})$ stages respectively, of response to cold treatment in plantain. While in cold-sensitive banana, no such equivalent regulation for ICE1-CBF-COR pathway was found and slow response to MYBS3 pathway responsible for the cold stress was observed. Thus, the low expression of the two key genes along with their delayed response to the cold stress may be one of the main reasons for cold sensitivity of banana. The cold-tolerant plantain also appears closely related to expressions of several specific genes such as CML7, AIR12 (for signal transduction), ZFP1, EIL1 (for transcriptional regulation), ELIP, CTL1, SCA, MKS1 (for stress response), MTP3 (for copper ion balance), RBCS (for photosynthesis and photorespiration), CP122 (sugar stimuli response) and $U B Q 11$ (protein modification). Combined with previous research, we rationalize that in early stage of cold stress response, changes in cell membrane phase trigger modification of actin cytoskeleton, which activates ICE1-CBF-COR metabolic pathways by $\mathrm{Ca}^{2+}$ and phosphorylation signaling pathways. Also it triggers an early response via the ethylene signal transduction pathway. In late stage of cold stress, expression of MYBS3 begins to recover. MYBS3 is involved in coordination with the expression of multiple genes for regulation of oxidation/reduction, oxylipin biosynthetic process, photosynthesis, photorespiration, glycolysis, tricarboxylic acid cycle, carbohydrate metabolic process, fatty acid biosynthetic process and beta-oxidation, enhanced adoption to cold tress (Figure 4). The next questions need to be answered are what causes the observed different expression profiles between ICE1 and MYBS3 pathways in plantain? How do both coordinate together for effectively regulating cold-resistance? Addressing these questions will further enhance the understanding on signaling and metabolic pathways of cold tolerance in plantain, but also conducive to nurturing new varieties of cold tolerant banana cultivars.

\section{Methods}

\section{Plant materials}

Seedlings of the cold-tolerant plantain (Musa spp. Dajiao; ABB Group) and the cold-sensitive banana (Musa spp. Cavendish; AAA Group) with a uniform growth stage were obtained from Institute of Fruit Tree Research, Guangdong Academy of Agricultural Sciences, Guangzhou, P. R. of China.

\section{Experimental design and cold stress treatment}

The main objective in this study was to compare coldresponse genes between cold-sensitive banana and coldtolerant plantain in order to gain a better understanding of the underlying signal transduction and the molecular mechanisms of cold tolerance plantain at transcriptomics level. Mature plantain plants can tolerate temperatures of $0-4^{\circ} \mathrm{C}$. In our previous quantitative proteomics analysis, we found 219 differentially expressed proteins in plantain seedlings with cold stress at $8^{\circ} \mathrm{C}$ for only 6 hours and the plant leaves drooped with wilting symptoms after $24 \mathrm{~h}$ of cold treatment [10]. To identify some sensitive and early cold-response genes in both banana and plantain, in this study we increased the cold- 


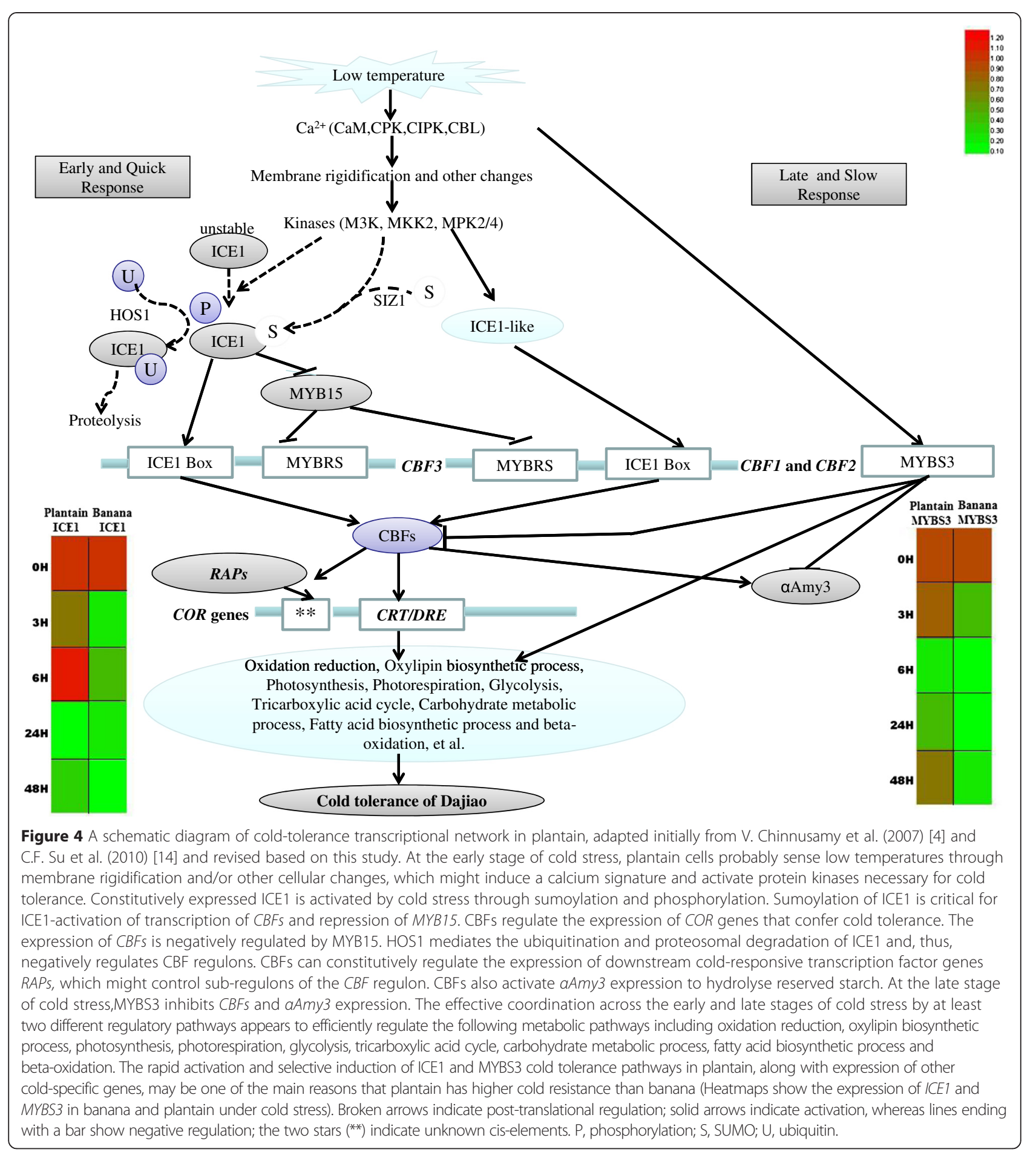

treatment temperature to $10^{\circ} \mathrm{C}$ and reduced exposure time at 0,3 and 6 hours respectively. The comparative transcriptomic analysis was conducted for the cold stressed seedlings, followed by large scale identification and functional categorization of the differentially expressed, early responsive genes. Furthermore, quantitative real time-PCR was carried out to validate the early cold-responsive transcriptomic results and some late-responsive genes with the extended time of cold treatment for 24 and 48 hours.

Seedlings were grown in a growth chamber at $30 / 28^{\circ} \mathrm{C}$ (day/night), a photon flux density of $240 \mu \mathrm{mol} \mathrm{m} \mathrm{m}^{-2} \mathrm{~s}^{-1}$ throughout a 12-h photoperiod, and a relative humidity of $60-80 \%$. Six-leaf stage seedlings were used in the 
experiment. Low temperature treatments were started at 12:00 AM on the first day by setting the temperature to $10^{\circ} \mathrm{C}$, which was reached about $30 \mathrm{~min}$ later. The first young leaf was detached from the top of each of the 5 plants at each time point $\left(10^{\circ} \mathrm{C}\right.$ for 0,3 and $\left.6 \mathrm{~h}\right)$ for each biological replicate. The leaves from the 5 plants were cut into pieces $(1.5 \times 1.5 \mathrm{~cm})$ and mixed well. Aliquots of the mixed tissues were frozen in liquid $\mathrm{N}_{2}$ and stored at $-80^{\circ} \mathrm{C}$ until use.

\section{Measurements of relative electrolyte leakage}

The leaves were cut into $1-\mathrm{cm}$ segments and washed three times with ultrapure water. The segments were placed in tubes containing $5 \mathrm{~mL}$ of ultrapure water and incubated at $25^{\circ} \mathrm{C}$. Two hours later, the electrical conductivity of the bathing solution (L1) was measured. Then the tubes were incubated at $100^{\circ} \mathrm{C}$ for $20 \mathrm{~min}$ and subsequently at $25^{\circ} \mathrm{C}$ for $1 \mathrm{~h}$, and the electrical conductivity (L2) was measured again. The relative electrolyte leakage was calculated by the formula $(L 1-L O) /(L 2-L O) \times$ 100 (L0, conductivity of ultrapure water) [53]. Five replicates were performed for each sample.

\section{Sample preparation, cDNA library construction and illumina sequencing}

Total RNA was extracted from banana and plantain leaves (Four biological replicates of Cavendish and Dajiao seedlings at $10^{\circ} \mathrm{C}$ for 0,3 and $6 \mathrm{~h}$ ) using plant total RNA isolation kit (Tiandz Inc; Beijing, China). RNA degradation and contamination was monitored on $1 \%$ agarose gels; RNA purity was checked using the NanoPhotometer ${ }^{\curvearrowleft}$ spectrophotometer (IMPLEN, CA, USA) and RNA integrity was assessed using the Bioanalyzer 2100 system (Agilent Technologies, CA, USA). A total of $3 \mu \mathrm{g}$ RNA per sample was used as input material in RNA sample preparations for subsequent cDNA library construction. All 24 samples had RIN values above 8.0. Sequencing libraries were generated using Illumina TruSeq $^{\mathrm{Tm}}$ RNA Sample Preparation Kit (Illumina, San Diego, USA) following manufacturer's recommendations and four index codes were added to attribute sequences to each sample. Briefly, mRNA was purified from total RNA using poly-T oligo-attached magnetic beads. Fragmentation was carried out using divalent cations under elevated temperature in Illumina proprietary fragmentation buffer. First strand cDNA was synthesized using random oligonucleotides and SuperScript II. Second strand cDNA synthesis was subsequently performed using DNA Polymerase I and RNase H. Remaining overhangs were converted into blunt ends via exonuclease/ polymerase activities and enzymes were removed. After adenylation of 3 ' ends of DNA fragments, Illumina PE adapter oligonucleotides were ligated to prepare for hybridization. In order to select cDNA fragments of preferentially $200 \mathrm{bp}$ in length the library fragments were purified with AMPure XP system (Beckman Coulter, Beverly, USA). DNA fragments with ligated adaptor molecules on both ends were selectively enriched using Illumina PCR Primer Cocktail in a 10 cycle PCR reaction. Products were purified (AMPure XP system) and quantified using the Agilent high sensitivity DNA assay on the Agilent Bioanalyzer 2100 system. The clustering of the index-coded samples was performed on a cBot Cluster Generation System using TruSeq PE Cluster Kit v3-cBotHS (Illumina) according to the manufacturer's instructions. After cluster generation, the library preparations were sequenced on an Illumina Hiseq 2000 platform and $100 \mathrm{bp}$ fragment reads were generated. The data was submitted into the NCBI SRA database (Accession No. SRP047347).

\section{Sequence annotation}

Raw data (raw reads) of fastq format were firstly processed through in-house perl scripts. In this step, clean data (clean reads) were obtained by removing reads containing adapter, reads containing ploy- $\mathrm{N}$, and low quality reads from raw data. At the same time, Q20, Q30, GC content and sequence duplication level of the clean data were calculated. All the downstream analyses were based on the clean data with high quality. Reference genome and gene model annotation files were downloaded from the banana genome website (http://banana-genome.cirad. $\mathrm{fr} /$ content/download-dh-pahang) directly. Index of the reference genome was built using Bowtie v0.12.8 and pairedend clean reads were aligned to the reference genome using TopHat v1.4.0. HTSeq v0.5.3 was used to count the reads numbers mapped to each gene. And then RPKM of each gene was calculated based on the length of the gene and reads count mapped to this gene.

\section{Identification of Differentially Expressed Genes (DEGs)}

Differential expression analysis was performed using the DEGSeq $R$ package (1.12.0). $P$-values were adjusted using the Benjamini \& Hochberg method. Corrected $P$-value of 0.05 and log2 (fold change) of 1 were set as the threshold for significantly differential expression. Gene Ontology (GO) enrichment analysis of differentially expressed genes was implemented by the GOseq $\mathrm{R}$ package, in which gene length bias was corrected. GO terms with corrected $P$-value less than 0.05 were considered significantly enriched by differential expressed genes.

\section{Quantitative RT-PCR}

Total RNA was isolated from banana and plantain leaves in four biological replicates at five different time points as described above. The resulting RNA $(1 \mu \mathrm{g})$ was used as a template for first-strand cDNA synthesis using ReverTra Ace (Toyobo, Osaka, Japan) with random 
hexamers according to the manufacturer's instructions. Primer pairs for real-time quantitative PCR (see Additional file 5: Table S5 online) were designed using Primer Premier 5.0 (Premier Biosoft, Palo Alto, USA). The PCR reaction consisted of $10 \mu \mathrm{L}$ of $2 \times$ SYBR Green PCR Master Mix (Toyobo), $200 \mathrm{nM}$ primers, and $2 \mu \mathrm{L}$ of 1:40diluted template cDNA in a total volume of $20 \mu \mathrm{L}$. No template controls were also set for each primer pair. Realtime PCR was performed employing the DNA Engine Option 2 Real-Time PCR Detection system and Opticon Monitor software (Bio-Rad, USA).

\section{Availability of supporting data}

The sequencing raw data of this article have been deposited in a SRA database at the NCBI -http://www.ncbi.nlm. nih.gov/Traces/sra_sub/sub.cgi?subid=321444\&from=list \&action=show:submission.

\section{Additional files}

Additional file 1: Table S1. The raw data quality of libraries.

Additional file 2: Table S2. Original data of Banana gene expression profiles under cold stress.

Additional file 3: Table S3. Original data of Plantain gene expression profiles under cold stress.

Additional file 4: Table S4. Primary functional classification on differential genes of Banana with fold changes $>$ or $<2$-fold.

Additional file 5: Table S5. List of primers used for the Real-time PCR.

Additional file 6: Figure S1. Relative mRNA levels of E2 SUMO-protein ligase SIZ1 in banana and plantain seedlings were determined by quantitative RT-PCR analyses. Six-leaf stage seedlings were incubated at $10^{\circ} \mathrm{C}$ for the indicated time. Transcript abundances of genes encoding E2 SUMO-protein ligase SIZ1 from both banana and plantain were determined and compared across the time course of cold stress. Data represent means \pm SD $(n=4)$. The different lowercase letters labeled above columns indicate a significant difference at $p \leq 0.05$ between the columns by Duncan's test using SPSS statistical software (version 16.0, SPSS Inc. Chicago, IL). The columns with the same letters mean no significant difference $(p>0.05)$ between each other.

\section{Abbreviations}

RPKM: Reads per kilobase of exon model per million mapped reads; DEG: Differentially expressed gene; GO: Gene ontology; ICE1: Inducer of CBF expression; CBF/DREB1: C-repeat/dehydration-responsive element binding factor; MYBS3: DNA-binding repeat MYB transcription factor S3; RCl: Rare cold-induced gene; ELIP: Early light-induced protein; CTL1: Chitinase-like protein 1; SCA: Stigma/stylar cysteine-rich adhesion; MKS1: MAP kinase substrate 1; MTP3: Metallothionein-like protein type 3; RBCS: Ribulose bisphosphate carboxylase small chain; CP122: Calvin cycle protein CP12-2; AIR12: Auxin-induced in root cultures protein 12; UBQ11: Polyubiquitin 11; GAPDH: Glyceraldehyde-3-phosphate dehydrogenase;

PRK: phosphoribulokinase; SUMO: Small ubiquitin related modifier; SOD: Superoxide dismutase; MDA: Malondialdehyde; ROS: Reactive oxygen species; RIN: RNA Integrity Number; ERF: Ethylene-responsive transcription factor; CBL: Calcineurin B-like protein; CIPK: CBL-interacting serine/threonineprotein kinase; $C M L$ : Calmodulin-like protein; $C O R$ : Cold regulated gene; EIL: Ethylene insensitive 3-like 1 protein; ADO: Adagio-like protein; STHY: Probable salt tolerance-like protein; ZFP: Zinc finger protein; Thi1-2: Thiamine thiazole synthase 2; GRP: Glycine-rich RNA-binding protein; DRT100: DNA-damage-repair/toleration protein DRT100; MPK4: Mitogenactivated protein kinases 4; MAP3K: MAP kinase kinase kinase; HOS1: RINGfinger E3 ubiquitin ligase; a-Amy3: a-amylase3.

\section{Competing interests}

The authors declare that they have no competing interests.

\section{Authors' contributions}

QY and GY conceived and designed the study. QY and JG wrote the manuscript. JG, WH and JW cultivated the banana plantlets, extracted RNA and participated in the bioinformatics analysis. SZ, TD, LD, CL, XP drafted the manuscript, designed the tables, critically reviewed the manuscript, and provided guidance. All authors read and approved the final manuscript.

\section{Acknowledgments}

This work was supported by National Natural Science Foundation of China No. 31201589 and 31372018; National High-tech R\&D Program (863 Program) No. 2011AA10020602; 948 Project from Ministry of Agriculture of China No. 2011-G16; National Banana Industry and Technology System Project No. CARS-32-01; International Collaborative Project from Ministry of Science and Technology of China No. 2013DFB30400; Commonwealth Industry (Agriculture) Specific Fund No. 200903049-10. The authors would like to thank Drs. Peter Schweitzer and Ted Thannhauser for their critical reading of this manuscript and helpful comments.

\section{Author details}

${ }^{1}$ Institute of Fruit Tree Research, Guangdong Academy of Agricultural Sciences, 80 Dafeng 2nd street, Tianhe District, Guangzhou, Guangdong Province 510640, China. ${ }^{2}$ Key Laboratory of South Subtropical Fruit Biology and Genetic Resource Utilization, Ministry of Agriculture, Guangzhou 510640, China. ${ }^{3}$ State Key Laboratory for Conservation and Utilization of Subtropical Agro-bioresources, South China Agricultural University, Guangzhou 510640, China. ${ }^{4}$ National Key Laboratory of Crop Genetic Improvement, Huazhong Agricultural University, Wuhan 430070, China. ${ }^{5}$ Institute of Biotechnology, Cornell University, Ithaca, NY 14853-2703, USA.

Received: 7 January 2015 Accepted: 17 April 2015

Published online: 10 June 2015

\section{References}

1. Zhou MQ, Shen C, Wu LH, Tang KX, Lin J. CBF-dependent signaling pathway: A key responder to low temperature stress in plants. Crit Rev Biotechnol. 2011;31(2):186-92.

2. Sanghera GS, Wani SH, Hussain W, Singh NB. Engineering cold stress tolerance in crop plants. Curr Genomics. 2011;12(1):30-43.

3. Nakashima K, Yamaguchi-Shinozaki K. Regulons involved in osmotic stressresponsive and cold stress-responsive gene expression in plants. Physiol Plant. 2006;126(1):62-71.

4. Chinnusamy V, Zhu J, Zhu J. Cold stress regulation of gene expression in plants. Trends Plant Sci. 2007;12(10):444-51.

5. Zhang T, Zhao X, Wang W, Pan Y, Huang L, Liu X, et al. Comparative Transcriptome Profiling of Chilling Stress Responsiveness in Two Contrasting Rice Genotypes. PLoS One. 2012;7(8):e43274.

6. Liu H, Ouyang B, Zhang J, Wang T, Li H, Zhang Y, et al. Differential Modulation of Photosynthesis, Signaling, and Transcriptional Regulation between Tolerant and Sensitive Tomato Genotypes under Cold Stress. PLoS One. 2012;7(11):e50785.

7. Xin H, Zhu W, Wang L, Xiang Y, Fang L, Li J, et al. Genome Wide Transcriptional Profile Analysis of Vitis amurensis and Vitis vinifera in Response to Cold Stress. PLoS One. 2013;8(3):e58740.

8. Zhang JZ, Creelman RA, Zhu JK. From laboratory to field. Using information from Arabidopsis to engineer salt, cold, and drought tolerance in crops. Plant Physiol. 2004;135(2):615-21.

9. D Hont A, Denoeud F, Aury J, Baurens F, Carreel F, Garsmeur O, et al. The banana (Musa acuminata) genome and the evolution of monocotyledonous plants. Nature. 2012;488(7410):213-7.

10. Yang QS, Wu JH, Li CY, Wei YR, Sheng O, Hu CH, et al. Quantitative proteomic analysis reveals that antioxidation mechanisms contribute to cold tolerance in plantain (Musa paradisiaca L.; ABB Group) seedlings. Mol Cell Proteomics. 2012;11(12):1853-69.

11. Israeli Y, Lahav E. Injuries to banana caused by adverse climate and weather. Diseases of Banana, Abacá Enset. CABI Publishing, Wallingford, Oxon, UK.2000, 351-379.

12. Lü Q F, Feng F, Zhang $X Z$ Z. Cold tolerance of banana in relation to cell structure in leaf tissue. J. Zhanjiang Ocean Univ, 2000, 20:48-51. 
13. Kai L, Chun-hua HU, DU Fa-xiu, Yu-e Z, Yue-rong W, Gan-jun YI. Over-Expression of the Arabidopsis CBF1 Gene in Dongguandajiao (Musa spp. ABB group) and Detection of Its Cold Resistance. Scientia Agricultura Sinica. 2012, 45(8):1653-1660.

14. Su CF, Wang YC, Hsieh TH, Lu CA, Tseng TH, Yu SM. A Novel MYBS3Dependent Pathway Confers Cold Tolerance in Rice. Plant Physiol. 2010;153(1):145-58.

15. Ma Y, Dai X, Xu Y, Luo W, Zheng X, Zeng D, et al. COLD1 Confers Chilling Tolerance in Rice. Cell. 2015, 160(6):1209-1221.

16. Zhang Q, Zhang JZ, Chow WS, Sun LL, Chen JW, Chen YJ, et al. The influence of low temperature on photosynthesis and antioxidant enzymes in sensitive banana and tolerant plantain (Musa sp.) cultivars. Photosynthetica. 2011;49(2):201-8.

17. Feng D, Liu B, Li W, He Y, Qi K, Wang JWH. Over-expression of a coldinduced plasma membrane protein gene ( $\mathrm{MpRCl}$ ) from plantain enhances low temperature-resistance in transgenic tobacco. Environ Exp Bot. 2009;65(2):395-402.

18. Zhang G, Guo G, Hu X, Zhang Y, Li Q, Li R, et al. Deep RNA sequencing at single base-pair resolution reveals high complexity of the rice transcriptome. Genome Res. 2010;20(5):646-54

19. Li W, Wu J, Zhang H, Shi S, Liu L, Shu B, et al. De Novo Assembly and Characterization of Pericarp Transcriptome and Identification of Candidate Genes Mediating Fruit Cracking in Litchi chinensis Sonn. Int J Mol Sci. 2014;15(10):17667-85.

20. Lu X, Kim H, Zhong S, Chen H, Hu Z, Zhou B. De novo transcriptome assembly for rudimentary leaves in Litchi chinesis Sonn. and identification of differentially expressed genes in response to reactive oxygen species. BMC Genomics. 2014, 15(1):805.

21. Luria N, Sela N, Yaari M, Feygenberg O, Kobiler I, Lers A, et al. De-novo assembly of mango fruit peel transcriptome reveals mechanisms of mango response to hot water treatment. BMC Genomics. 2014;15(1):957.

22. Urasaki N, Tarora K, Shudo A, Ueno H, Tamaki M, Miyagi N, et al. Digital Transcriptome Analysis of Putative Sex-Determination Genes in Papaya (Carica papaya). PLoS One. 2012;7(7):e40904

23. Crifò T, Puglisi I, Petrone G, Recupero GR, Lo Piero AR. Expression analysis in response to low temperature stress in blood oranges: Implication of the flavonoid biosynthetic pathway. Gene. 2011;476(1-2):1-9.

24. Perrone I, Pagliarani C, Lovisolo C, Chitarra W, Roman F, Schubert A Recovery from water stress affects grape leaf petiole transcriptome. Planta. 2012;235(6):1383-96

25. VAN DEN, BERG N, BERGER DK, HEIN I, BIRCH PRJ, WINGFIELD MJ, et al. Tolerance in banana to Fusarium wilt is associated with early up-regulation of cell wall-strengthening genes in the roots. Mol Plant Pathol. 2007;8(3):333-41.

26. Chinnusamy V, Ohta M, Kanrar S, Lee BH, Hong X, Agarwal M, et al. ICE1: a regulator of cold-induced transcriptome and freezing tolerance in Arabidopsis. Genes Dev. 2003;17(8):1043-54.

27. Lu CA, Ho TH, Ho SL, YU SM. Three novel MYB proteins with one DNA binding repeat mediate sugar and hormone regulation of alpha-amylase gene expression. Plant Cell. 2002;14(8):1963-80.

28. Ruelland E, Vaultier M, Zachowski A, Hurry V. Cold Signaling and Cold Acclimation in Plants. Advances in Botanical Research. 2009, 49:35-150.

29. Ding Y, Li H, Zhang X, Xie Q, Gong Z, Yang S. OST1 Kinase Modulates Freezing Tolerance by Enhancing ICE1 Stability in Arabidopsis. Dev Cell. 2015;32(3):278-89.

30. Miura K, Ohta M, Nakazawa M, Ono M, Hasegawa PM. ICE1 Ser403 is necessary for protein stabilization and regulation of cold signaling and tolerance. Plant J. 2011:67(2):269-79.

31. Saijo Y, Hata S, Kyozuka J, Shimamoto K, Izui K. Over-expression of a single $\mathrm{Ca}^{2+}$-dependent protein kinase confers both cold and salt/drought tolerance on rice plants. Plant J. 2000;23(3):319-27.

32. Xu G, Rocha PSCF, Wang M, Xu M, Cui Y, Li L, et al. A novel rice calmodulinlike gene, OSMSR2, enhances drought and salt tolerance and increases ABA sensitivity in Arabidopsis. Planta. 2011;234(1):47-59.

33. Du H, Liu H, Xiong L. Endogenous auxin and jasmonic acid levels are differentially modulated by abiotic stresses in rice. Front Plant Sci. 2013;4:397.

34. Neuteboom LW, Ng JM, Kuyper M, Clijdesdale OR, Hooykaas PJ, van der Zaal BJ. Isolation and characterization of CDNA clones corresponding with mRNAs that accumulate during auxin-induced lateral root formation. Plant Mol Biol. 1999:39(2):273-87.
35. Rohde P, Hincha DK, Heyer AG. Heterosis in the freezing tolerance of crosses between two Arabidopsis thaliana accessions (Columbia-0 and C24) that show differences in non-acclimated and acclimated freezing tolerance. Plant J. 2004:38(5):790-9.

36. Lo Piero AR, Lo Cicero L, Puglisi I. The metabolic fate of citric acid as affected by cold storage in blood oranges. J Plant Biochem Biot. 2014;23(2):161-6.

37. Hu H, You J, Fang Y, Zhu X, Qi Z, Xiong L. Characterization of transcription factor gene SNAC2 conferring cold and salt tolerance in rice. Plant Mol Biol. 2008:67(1-2):169-81.

38. Zhang Z, Huang R. Enhanced tolerance to freezing in tobacco and tomato overexpressing transcription factor TERF2/LeERF2 is modulated by ethylene biosynthesis. Plant Mol Biol. 2010;73(3):241-9.

39. Zhang $Y$, Chen $C$, Jin XF, Xiong AS, Peng RH, Hong $Y H$, et al. Expression of a rice DREB1 gene, OsDREB1D, enhances cold and high-salt tolerance in transgenic Arabidopsis. BMB Rep. 2009;42(8):486-92.

40. Agarwal M, Hao Y, Kapoor A, Dong CH, Fujii H, Zheng X, et al. A R2R3 Type MYB Transcription Factor Is Involved in the Cold Regulation of CBF Genes and in Acquired Freezing Tolerance. J Biol Chem. 2006;281(49):37636-45.

41. Peng Y, Bartley LE, Canlas P, Ronald PC. OsWRKY Ila Transcription Factors Modulate Rice Innate Immunity. Rice. 2010;3(1):36-42.

42. Wang Q, Wang M, Zhang X, Hao B, Kaushik SK, Pan Y. WRKY gene family evolution in Arabidopsis thaliana. Genetica. 2011;139(8):973-83.

43. Jakoby M, Weisshaar B, Dr Ge-Laser W, Vicente-Carbajosa J, Tiedemann J, Kroj TPF. bZIP transcription factors in Arabidopsis. Trends Plant Sci. 2002;7(3):106-11.

44. Yu G, Jiang L, Ma X, Xu Z, Liu M, Shan S, et al. A Soybean C2H2-Type Zinc Finger Gene GmZF1 Enhanced Cold Tolerance in Transgenic Arabidopsis. PLoS One. 2014;9(10):e109399.

45. Boutrot F, Segonzac C, Chang KN, Qiao H, Ecker JR, Zipfel C, et al. Direct transcriptional control of the Arabidopsis immune receptor FLS2 by the ethylene-dependent transcription factors EIN3 and EIL1. Proc Natl Acad Sci U S A. 2010;107(32):14502-7.

46. Peng Y, Lin W, Wei H, Krebs SL, Arora R. Phylogenetic analysis and seasonal cold acclimation-associated expression of early light-induced protein genes of Rhododendron catawbiense. Physiol Plant. 2007;132(1):44-52.

47. Hermans C, Porco S, Verbruggen N, Bush DR. Chitinase-Like Protein CTL1 Plays a Role in Altering Root System Architecture in Response to Multiple Environmental Conditions. Plant Physiol. 2010;152(2):904-17.

48. Andreasson E, Jenkins T, Brodersen P, Thorgrimsen S, Petersen NHT, Zhu S, et al. The MAP kinase substrate MKS1 is a regulator of plant defense responses. EMBO J. 2005:24(14):2579-89.

49. Zhang M, Takano T, Liu S, Zhang X. Abiotic stress response in yeast and metal-binding ability of a type 2 metallothionein-like protein (PutMT2) from Puccinellia tenuiflora. Mol Biol Rep. 2014;41(9):5839-49.

50. Izumi M, Tsunoda H, Suzuki Y, Makino A, Ishida H. RBCS1A and RBCS3B, two major members within the Arabidopsis RBCS multigene family, function to yield sufficient Rubisco content for leaf photosynthetic capacity. J Exp Bot. 2012;63(5):2159-70.

51. Marri L, Pesaresi A, Valerio C, Lamba D, Pupillo $P$, Trost $P$, et al. In vitro characterization of Arabidopsis CP12 isoforms reveals common biochemical and molecular properties. J Plant Physiol. 2010;167(12):939-50

52. von Kampen J, Nieländer U, Wettern M. Expression of ubiquitin genes in Chlamydomonas reinhardtii: involvement in stress response and cell cycle. Planta. 1995;197(3):528-34.

53. Ai-kui C, Rui-hong H, Dong-yang LI, Lian-lian L, Hui-xia L, Shang-jian T. A Comparison of Two Methods for Electrical Conductivity about Plant Leaves. J Guangdong Educ Inst. 2010, 5:018. 